\title{
La structure du poème de Parménide
}

\section{Livio Rossetti}

\section{(2) OpenEdition}

\section{Journals}

Édition électronique

URL : https://journals.openedition.org/philosant/2318

DOI : 10.4000/philosant.2318

ISSN : 2648-2789

\section{Éditeur}

Éditions Vrin

\section{Édition imprimée}

Date de publication : 30 octobre 2010

Pagination : 187-226

ISBN : 978-2-7574-0179-8

ISSN : $1634-456$

\section{Référence électronique}

Livio Rossetti, «La structure du poème de Parménide », Philosophie antique [En ligne], 10 | 2010, mis en ligne le 11 juillet 2019, consulté le 02 décembre 2022. URL : http://journals.openedition.org/philosant/ 2318 ; DOI : https://doi.org/10.4000/philosant.2318

\section{$\Theta \oplus \Theta \Theta$}

Creative Commons - Attribution - Pas d'Utilisation Commerciale - Pas de Modification 4.0 International - CC BY-NC-ND 4.0

https://creativecommons.org/licenses/by-nc-nd/4.0/ 


\title{
LA STRUCTURE DU POÈME DE PARMÉNIDE
}

\author{
Livio ROSSETTI
}

Université de Pérouse

RÉSUMÉ. La structure qui sous-tend la composition du poème de Parménide est très élaborée, il est aisé de s'en rendre compte. La déesse y parle des enseignements qu'elle s'apprête à délivrer et, dans les fragments 10 et 11, elle offre un panorama détaillé de tout un ensemble de questions qu'elle va traiter aussitôt après. Un certain nombre d'éléments métatextuels se trouvent de cette façon insérés dans le texte écrit au premier degré et en interrompent le cours. D'autres passages de texte à métatexte (et vice versa) se rencontrent d'ailleurs dans les fragments, mettant ainsi en évidence de frappantes discontinuités et des changements significatifs dans le type de saturation provoqué dans l'auditoire. Tout cela fait comprendre que les enseignements délivrés dans le poème sont immergés dans une infrastructure beaucoup plus « construite » et «étudiée », beaucoup plus consciente que celle qui apparaît dans tant d'autres textes de la même époque ou antérieurs. D'où l'importance d'une recherche spécifiquement consacrée à la composition du poème dans son ensemble.

La formule rassurante de B10.1, «tu apprendras » ( $\left.\varepsilon \iota^{\prime} \sigma \eta\right)$, prend dans cette recherche une importance particulière, parce qu'elle démontre que Parménide lui-même a donné une valeur explicitement positive à ce qui, jusque peu auparavant, faisait l'objet de jugements fortement négatifs. Cette incohérence n'est donc pas une conjecture de notre part, mais quelque chose dont Parménide ne peut pas ne pas s'être rendu compte, ce qui signifie vraisemblablement que la seconde partie du poème expose des connaissances auxquelles il était parvenu dans une «phase précritique » de sa recherche. Sinon, à quelle autre explication pourrait-on penser?

SUMMARY. It is quite easy to perceive that Parmenides' poem is governed by a complex compositional 'architecture'. His goddess speaks of the teachings (plural) she is going to offer and, in frgs. 10-11, gives a detailed outline of the themes to be treated in the sequel. Thus some meta-texts happen to be entered into the first order text and break its continuity. However the fragments give evidence of further passages from text to meta-text (and vice versa), as well as of a clear discontinuity from section to section. They also show remarkable changes in the sort of saturation instilled into the audience. For all these reasons, the doctrines offered in the poem appear to be involved in a much more structured, more learned, more conscious frame than 
what emerges from contemporary or earlier writings. A research project especially devoted to the compositional structure of the poem as a whole was therefore worth being undertaken.

Within this investigation, a special attention is being paid to the reassuring "you'll learn" (Elon) of B10.1, since it shows that Parmenides himself came to value positively what had been previously charged with heavy negative evaluations. Therefore, this incongruence is not a conjecture but something Parmenides was necessarily aware of. This, in turn, is likely to mean that the second part of the poem contains the sort of knowledge he had attained during a "precritical stage' of his research. Otherwise, which other explanation could be devised? 


\section{Un poème hyper-structuré et un auteur tout à fait conscient de ce qu'il écrit}

Que le poème de Parménide soit régi par une structure très élaborée et innovante, il est aisé de s'en rendre compte. Pensons seulement au fait que la déesse, au moment où elle prend la parole, s'empresse non seulement d'annoncer deux logoi différents, mais aussi de préciser que ces logoi ne revendiquent pas du tout la même crédibilité : haute ou très haute dans le cas du discours sur l'être, très inférieure (mais seulement inférieure, puisqu'il est cependant destiné à être enseigné et appris) dans le cas du discours sur le cosmos et le monde de la vie. Nous avons ensuite la mémorable formule de transition qui sépare le premier logos du second (B8, 50-52) : une formule qui, tout en respectant parfaitement l'annonce de deux enseignements, non seulement trace la frontière entre eux, mais fait aussi en sorte d'indiquer à nouveau les différences qu'ils présentent quant à leur crédibilité respective (c'est-à-dire à ce qu'on appelle leur coefficient épistémique). Même le second logos, en effet, bien qu'il soit composé de simples «opinions de mortels » et donc par définition d'une infériorité incommensurable vis-à-vis de la vérité, n'est pas simplement nécessaire aux mortels, mais se distingue du savoir commun des mortels :

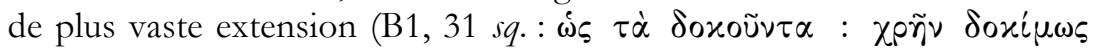

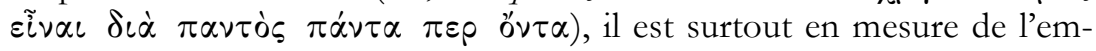
porter sur n'importe quelle autre doxa ( $f f . \mathrm{B} 8,61: \dot{\omega} \varsigma$ oủ $\mu \dot{n} \pi \circ \tau \varepsilon \tau i \varsigma$

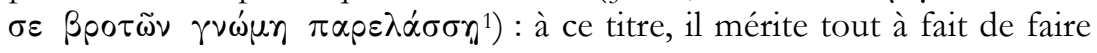
l'objet d'un enseignement non dépourvu d'autorité, enseignement que, précisément, la déesse se propose de délivrer aussitôt après.

1. Je cite d'après l'édition de Cerri 1999. 
Figure en outre - vraisemblablement dans le contexte du second lo$g o s^{2}-$ l'annonce des thèmes qui seront abordés dans la suite (fr. 10 et 11), annonce qui prend la forme d'un panorama détaillé de toute une série de sujets qui seront traités sous la forme d'enseignements plus approfondis. Et puisqu'il y a des indices certains de la présence, dans le poème, d'autres sous-sections à caractère naturaliste, il est au moins possible que l'annonce formulée dans les fragments 10 et 11 veuille renvoyer, non à la totalité du second logos, mais seulement à une partie du vaste savoir peri physeos qui était proposé à part du discours sur l'être (le cœur de l'enseignement de la déesse). En particulier les deux fragments qui portent sur le sexe de l'enfant à naître touchent à une question si spécifique qu'on est amené à penser qu'elle faisait partie d'un iatrikos logos plus détaillé. Ce fait, à son tour, ouvre la porte à l'éventualité que d'autres soussections aient été aussi introduites en temps opportun, telle celle relative aux corps célestes, avec une formule de transition spécifique (un intermède) à caractère métadiscursif qui annonçait la sous-section suivante. J'observe enfin que le fragment 19 se présente comme la conclusion du second logos, voire de tout le discours de la déesse. Après semblable conclusion, il est au moins possible que le poète-philosophe reprenne la narration en première personne et que, une fois conclu le discours de la déesse, la narration se poursuive brièvement en faisant place à un remerciement de la part du kouros et, pour finir, à une sphragis guère différente de celle utilisée par Thucydide (p. ex. «Voici ce qu'a dit Parménide fils de Pyrès », ou "Voici ce qu'a dit la déesse à Parménide fils de Pyrès »).

Grâce à la présence d'un si grand nombre d'indices indubitablement métadiscursifs, on voit se dessiner une organisation sophistiquée du texte poétique en sections, comportant une alternance réglée d'unités discursives et méta-discursives, et un locuteur qui, en diverses occasions, prend soin d'identifier et de caractériser ce qui est sur le point d'être enseigné dans la suite (ainsi, éventuellement, que ce qui vient d'être exposé). Les commentateurs rappellent volontiers, à ce sujet, quelques vers d'Hésiode

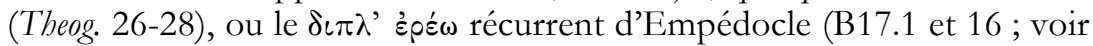
aussi B8.1), mais les différences sautent aux yeux. Les Muses d'Hésiode, quand elles commencent en parlant de ce qu'elles s'apprêtent à chanter, évoquent l'ambivalence structurelle de leur chant, qui fait coexister fonction didactique et fonction de divertissement et de libre construction fan-

2. Cordero a essayé récemment de contester ce point: d'après lui, l'exposé cosmologique, le iatrikos logos et éventuellement d'autres enseignements positifs devraient faire partie du développement sur la vérité. Cette thèse a été sommairement esquissée dans Cordero et al. 2009, p. 78-80, et l'on en attend une exposition dûment argumentée dans une autre publication. On peut dire cependant que cette hypothèse va à l'encontre de déclarations de la déesse explicites et répétées. 
tastique. Or cela revient à dire que chez Hésiode ces deux composantes coexistent et ne peuvent pas ne pas coexister (inoffensif truisme). À l'inverse, Parménide distingue, identifie ce qu'a de spécifique chaque partie de son chant, il identifie des sous-domaines et trouve le moyen de parler de la spécificité de chaque section et même de l'ordre dans lequel sont traités les différents thèmes. En outre le fragment 8 fournit des indices substantiels qui font penser qu'il sait tenir ses promesses, évitant de mêler et de confondre les registres de son discours. Cela est même tout simplement extraordinaire, car Parménide fournit ainsi à plusieurs reprises des indications précises sur les principes d'organisation de son poème. Dans ce but, il emploie plusieurs vers, prenant soin de distinguer les sujets déjà traités de ceux qui n'ont pas encore été abordés et, comme je le disais, allant en outre jusqu'à signaler la valeur cognitive propre à chaque sous-unité au sein de l'ensemble. En procédant ainsi, le poètesophos trouve en outre le moyen de signaler que c'est tout à fait consciemment qu'il a introduit un tel ordonnancement et qu'il a la volonté de s'y tenir. C'est cette fois la comparaison avec Empédocle qui sert en quelque sorte de contre-épreuve, puisque ce dernier propose, comme on le sait, un continum substantiellement indifférencié où il y a place pour des choses qui peuvent être très diverses, comme celles qui procèdent d'une suggestion de la part du prophète-sauveur et des considérations compréhensibles, avec lesquelles on peut en gros être d'accord, sur des thèmes particuliers. Alors que le message conçu pour exercer une suggestion coexiste avec des passages clairement didactiques, le passage fréquent d'un registre à l'autre ne permet d'apercevoir ni critères, ni démarcations, ni morceaux qui aient une fonction métadiscursive manifeste. Ce n'est pas un hasard si la distinction entre Peri physeos et Katharmoi a été sérieusement mise en doute à la suite de la publication du Papyrus de Strasbourg. Dans le poème de Parménide, au contraire, prend tout son relief la nette discontinuité qui se produit quand l'auteur introduit un métatexte sous la forme d'une formule de transition qui non seulement raccorde et sépare à la fois ce qui précède et ce qui suit, mais se constitue comme une entité objectivement différente par comparaison tant avec le logos qui précède qu'avec celui qui suit. L'introduction récurrente de telles discontinuités constitue un trait particulier du poème de Parménide.

Outre le fait qu'ils nous offrent un nombre non négligeable de passages de texte à métatexte et vice versa, les fragments mettent en évidence d'incontestables discontinuités d'ordre tonal et thématique et même, comme j'espère le montrer, des indices d'une énergique modulation de l'horizon de saturation induit chez l'auditeur. Particulièrement voyant est le changement de tonalité que l'on observe quand la narration préliminaire cède la place aux déclarations programmatiques de la déesse qui 
donnent le coup d'envoi au développement sur la vérité. Dans ce cas, le caractère hautement fonctionnel du proème par rapport au développement qui lui fait suite n'implique pas une continuité compositionnelle par rapport aux logoi qui viennent après. On observe au contraire une profonde modification tant de la démarche que du style. C'est ce qu'a écrit en particulier Cerri :

La nouveauté du style de Parménide se démontre sans peine à partir d'une comparaison du fr. 1 et du fr. 7/8. Le premier, précisément parce qu'il est de contenu narratif, est encore très proche de la fluidité rythmico-syntaxique de l'ancienne diction épique. Le second qui, comme nous l'avons vu, est l'exposition d'un théorème, se caractérise au plus haut point par une âpreté dans l'expression qui brise continuellement le rythme ordinaire de l'hexamètre et concentre l'attention de l'auditeur sur chaque articulation du discours, sur chaque lexème, lesquels consistent souvent en néo-formations stupéfiantes qui soulignent la hardiesse des montages conceptuels ${ }^{3}$.

De fait, alors que le proème sait capter l'attention de l'auditeur-lecteur et parvient à l'immerger dans un monde de fantaisie qui, de toute évidence, sert à lancer le processus d'acclimatation aux enseignements qui vont suivre ${ }^{4}$, lui fait suite un enseignement proprement dit, proposé non plus selon une modalité narrative, comme une sorte de passe-temps ou un ensemble d'informations à enregistrer, mais comme un raisonnement que l'auditoire doit pouvoir comprendre et de la validité duquel il doit pouvoir se convaincre.

D’une façon qui n'est pas très différente, en B8.50-52, le choix de déclarer achevé un discours d'importance, ample et continu, pour annoncer de la façon la plus explicite le passage à un autre type de discours et à un horizon d'attente différent - ce choix répond à une multiplicité d'objectifs assez faciles à identifier :

- susciter des attentes nouvelles ;

- prévenir la confusion ;

3. Cerri 1999, p. 92.

4. Robbiano 2006 parle, non sans raison de «philosophie transformative » (voir infra p. 204), bien qu'en réalité la fonction transformative sous l'effet de laquelle l'auditoire docile se laisse transporter dans un monde indiscutablement différent de son univers familier (un monde régi par des règles nouvelles, incompatibles avec son vécu quotidien) soit une fonction éminemment (macro) rhétorique. Nous devons seulement garder à l'esprit que, à côté de l'affabulation dans l'art, il existe une capacité affabulatrice sui generis qui se manifeste dans les traités, dans les commentaires et, de façon plus générale, dans la construction du savoir (cf. Rossetti 1994). 
- aider l'auditoire à maintenir fermement la distinction entre le logos qui vient de s'achever et celui qui va commencer ;

- accompagner le passage d'une façon de raisonner à une autre (avec des différences qui à leur tour se situent à différents niveaux) ;

- confirmer l'opportunité de prêter grande attention aussi à ce qui va suivre, bien qu'il s'agisse d'un savoir très différent, et d'un savoir qui n'a pas la même sublimité.

Tout cela avec la conscience évidente, de la part du poète, de ce qu'il est en train de faire. Bref, le fait d'annoncer les deux logoi démontre que le très long monologue de la déesse ${ }^{5}$ est conçu à l'avance comme un ensemble constitué de parties qui sont à la fois reliées entre elles et distinguées les unes des autres par des formules de transition appropriées.

Une fonction analogue est assignée aux fragments 10-11, car dire «maintenant tu connaîtras la nature de l'air, les étoiles, le soleil, etc.» équivaut à dire «maintenant nous allons changer de sujet, à partir de maintenant nous parlerons d'une autre chose, elle aussi importante, ce pour quoi je t'invite à prêter attention à ces autres sujets »; ce qui veut dire que l'on ne passe pas d'une sous-section à une autre du même genre, comme si la différence d'objet ne touchait pas aussi au type d'exposé, comme si différentes sous-sections s'enchaînaient l'une après l'autre. Au contraire, dans le cas du fragment 19, la fonction récapitulatrice se combine avec une fonction interprétative. En effet, dans ce cas, la déesse rappelle, répète, souligne que «tout ce que j'ai dit jusque-là » (ou mieux : " ce qu'il m'est arrivé de dire au cours de mon second logos») est constitué d'affirmations faites $x \alpha \tau \dot{\alpha} \delta \delta^{\prime} \xi \alpha \nu$, se fonde sur des noms que les hommes ont institués, et n'a par conséquent qu'un statut épistémique peu élevé.

De là résulte que le très long monologue de la déesse constitue un ensemble structuré, régi par un principe d'ordre, avec des divisions et subdivisions dont chacune était clairement annoncée et dont était, à l'occasion, tirée la conclusion. Par conséquent les conditions sont réunies qui permettent de reconnaître dans le poème un projet communicationnel complexe, comportant la juxtaposition d'amples développements discursifs nettement différenciés les uns des autres, selon une logique de composition et un plan de construction : un projet communicationnel

5. Nous ne sommes pas en mesure d'estimer la longueur du poème de Parménide, mais nous pouvons en tout cas exclure que le nombre de vers puisse être inférieur à 500 . Quelle que soit la longueur totale du poème, il ne contenait en dehors du monologue que les vingt-trois premiers vers et, peut-être, une douzaine d'autres à la fin. En effet la déesse ne s'interrompt ni à la fin du prologue ni au moment de passer du premier au second logos. 
qui organise savamment la coexistence, pas toujours aisée, des différents discours en lesquels s'articule l'ensemble.

Ces caractéristiques invitent donc à parler d'une "architectonique » du poème, c'est-à-dire à reconnaître dans le poème un degré exceptionnellement élevé de visibilité des subdivisions dont il se compose, avec des indicateurs qui permettent d'identifier avec précision la fonction remplie par chaque subdivision. De manière indirecte, ces caractéristiques nous parlent de la conscience du poète qui a trouvé les façons appropriées d'identifier (et de nous aider à identifier) la fonction et les caractéristiques particulières de chacune des subdivisions de son œuvre. Ce que vise la présente étude, c'est précisément de mettre en évidence les aspects structurels ou architectoniques de l'œuvre; dans ce but, elle se propose tout d'abord de repérer chaque subdivision, ainsi que les indices d'une interaction fonctionnelle entre elles ${ }^{6}$.

Quiconque a commenté les fragments a au moins effleuré le sujet, mais on ne peut pas dire que se soit constituée une tradition d'études spécifiquement consacrées à l'«architectonique» du poème. En effet, pour prêter attention à sa structure portante, il faut en considérer l'ensemble et résister à l'attrait qu'exercent individuellement ses ingrédients, chose qui dans bien des cas n'a pas eu lieu du fait que certains de ces ingrédients - en particulier la doctrine de l'être et le proème - ont très puissamment, voire exclusivement polarisé l'attention des commentateurs. Par exemple, quand l'attention s'est portée plus particulièrement sur le proème, c'est la tendance à rechercher des indices de continuité avec la tradition épique qui a largement prévalu sur la disposition à relever aussi les indices de discontinuité, qui ne sont pourtant pas rares et loin d'être imperceptibles. Quand, ensuite, on s'est interrogé sur le sens à attribuer à la section doxastique du poème, étant donné que Parménide semble décidé à rabaisser cette section au rang d'un élément ouvertement secondaire et subalterne par rapport à l'ensemble, ce n'est pas sur l'ensemble de ce second enseignement que s'est focalisée l'attention des interprètes, mais sur les déclarations initiales à propos de cet enseignement, et sur la recherche d'une place raisonnable pour ce savoir de second rang en se plaçant dans l'optique du premier (chose qui, j'espère réussir à le démontrer, est proprement une erreur). En tout cas, un examen à nouveaux frais de l'architecture du poème permet de relever d'autres indices de l'acuité avec laquelle Parménide a visiblement eu conscience du problème posé par la coexistence des deux logoi et du point d'équilibre qu'il a

6. J'aurai l'occasion de montrer que l'attention portée aux aspects architectoniques aide aussi à mieux comprendre certains passages cruciaux, mais ce n'est pas là l'objectif premier de ma recherche. 
trouvé. Pourtant, on pense ordinairement que l'interprète ne peut que noter la présence encombrante d'un second développement, ample mais nettement inférieur à celui qui porte sur l'être, au point d'être inessentiel, non nécessaire et même superflu ${ }^{7}$, comme si Parménide n'avait pas contribué de manière significative à clarifier la situation ou n'en avait même pas eu conscience. J'en conclus que ces indices de la conscience qu'avait Parménide du problème, il fallait (il aurait fallu) en prendre acte avant d'élaborer des conjectures sur le sens du second logos et de la relation entre les deux types de savoir professés par la déesse.

Pour ces raisons entre autres, face à une polarisation générale de l'attention sur les données paléographiques, les détails textuels, les noyaux doctrinaux, les passages difficiles et autres questions particulières, il paraît souhaitable de réfléchir aussi aux nombreux indices révélateurs d'un projet, ou au moins aux passages qui jouent manifestement le rôle de liens et qui président au "montage» fonctionnel des parties dont se compose l'ensemble. La présente étude se propose précisément d'attirer l'attention sur les différents «morceaux» ou ingrédients de l'ensemble, de relever ce qui les différencie les uns des autres, pour finalement construire des hypothèses sur la fonction assignée à chaque «morceau » en même temps que sur l'articulation d'une telle multiplicité d'ingrédients. Prend forme de cette façon une approche systémique ou holistique du poème ${ }^{8}$, et on peut espérer que le tour ainsi donné à l'investigation puisse contribuer à jeter une lumière nouvelle sur l'ensemble.

\section{Le (premier) Proème}

Le poème s'ouvre sur une narration faite en première personne par le poète «du haut du char qu'il occupe » (Gálvez Garduño 2008) et qui se déploie dans les vingt-et-un premiers vers du fragment 1 (ou, si l'on préfère, jusqu'au premier colon du vers 28), sans comporter d'éléments de discontinuité interne significatifs.

Parmi les facteurs d'unité de cet ensemble, une place importante revient à l'évocation continuelle de sons. Grâce à eux prend forme une sorte de bande sonore qui accompagne la récitation ${ }^{9}$ : au début ce sont

7. Voir à ce sujet infra n. 15.

8. Sauf erreur de ma part, rares et, sans exception, récentes sont les contributions qui s'inspirent d'une telle approche. Je rappellerai ici Robbiano 2006 et Ramírez Vidal 2005, spécialement p. 85-120.

9. Faire état de cette sorte de bande sonore n'implique pas que l'on suppose une matérialité du voyage, mais seulement que Parménide pouvait rêver ou imaginer le voyage en char, vraisemblablement en faisant référence à un environnement concret assez sem- 
les roues qui font du bruit, les chevaux, et peut-être les Héliades, puis les paroles adressées à la déesse portière; ensuite le grincement de la serrure et de la porte, la reprise du parcours pour un autre court moment, les chevaux qui s'arrêtent, les gestes et les pas du kouros qui descend du char, et enfin la voix, à la fois solennelle et rassurante, de la déesse.

L'alternance de vague et de précision ${ }^{10}$ contribue à donner un peu de mouvement à la narration. Les cinq premiers vers, qui dessinent une situation absolument vague et floue, répondent vraisemblablement à l'intention d'éveiller la curiosité sans la satisfaire aussitôt. Le thymos évoque une poussée émotionnelle du locuteur sans donner aucun moyen de la décoder; de même, l'adjectif polyphemon nourrit des attentes que nous sommes incapables de préciser. Tout aussi vagues, il faut l'avouer, le kata pant' et la relative qui suit, ainsi que l'adjectif eidota et, au vers suivant, polyphrastoi. Tout se joue donc sur l'attente d'événements imminents, mais que l'auditeur n'a aucun moyen de se représenter. Aussitôt après viennent au contraire deux vers centrés sur l'observation minutieuse des effets du frottement sur l'essieu des roues. Suit une nouvelle présentation d'éléments narratifs qui ne se laissent pas décoder intuitivement (les filles du Soleil et la maison de la Nuit, l'arrivée à la porte, sa description sommaire, la déesse gardienne, les paroles doucereuses des Héliades), après quoi sont à nouveau présentées des données d'observation plus précises sur la serrure et les gonds de la grande porte (v. 17-20) ${ }^{11}$. Suit une nouvelle section narrative (l'arrivée à la demeure de la déesse, la poignée de mains, les paroles de bienvenue...), après quoi a lieu le "passage de témoin »: «la déesse m'accueillit, me prit la main et commença à parler. Elle me dit...» (v. 22-23). La possibilité est ainsi donnée au lecteur de noter le passage à un second locuteur.

Par delà les détails relevés ci-dessus, on reconnait facilement dans le prologue les traits caractéristiques du mythos: l'auditeur est plongé dans une situation fantastique à connotations positives, dans l'attente d'événements ou de signes qui lui permettent de préciser peu à peu le sens de cette situation inattendue, il est implicitement invité à suivre docilement la narration, à l'accepter sans se hâter de porter un jugement ni céder à la

blable à celui que l'on peut encore observer dans les fouilles d'Élée, en particulier à la route qui mène du port à la ligne de crête, au nord-est de la zone des temples.

10. «Sublime vagueness and painstaking realism alternate... a quite careful and deliberate structural scheme » (Mansfeld 1984, p. 6). Ramírez Vidal 2005, p. 106 sq., distingue cinq sections dans le proème (v. 1-5, 6-10, 11-14, 15-21 e 22-28a) et désigne cette dernière section comme "seconde partie du Proème ». Il semble inutile de s'arrêter sur les raisons de divergences de détail par rapport à la division proposée plus haut.

11. Rappelons l'important appendice "Ueber altgriechische Thüren und Schlösser » ajouté par Diels 1897. 
tentation de prendre du recul. Il s'apprête ainsi à laisser l'histoire modeler peu à peu son monde imaginaire, voire tout simplement son monde mental. Dans cette phase, en effet, l'auditeur-lecteur n'est pas invité à raisonner mais à libérer son imagination, à s'immerger docilement dans l'histoire et à éveiller sa curiosité. On ne lui demande rien d'autre, et d'ailleurs on ne le met pas en condition de faire autre chose.

Gálvez Garduño (2008, p. 67) a signalé « l'absence délibérée de la première personne... le pronom moi, qui se rencontre trois fois dans les cinq premiers vers, n'apparait pas une seule fois du vers 6 au vers $21 »$. Cette rapide atténuation de la référence du narrateur à lui-même a pour effet d'accentuer l'aspect objectif de la situation et ainsi facilite le processus d'identification de l'auditeur. À son tour la présence récurrente de connotations non intuitives sert à créer un halo de mystère et à accroître la curiosité. Parmi ces éléments non intuitifs se détache le recours à des paroles habiles pour surmonter l'obstacle que représente la grande porte : détail qui nous permet de deviner que les Héliades ont une mission à remplir et que Dike représente pour elles un problème à résoudre.

Par rapport aux conventions de la poésie épique, il est aisé de constater que, dans ce cas, le protagoniste de l'histoire racontée est le poète lui-même, qui n'hésite pas à s'attribuer une condition plus que privilégiée, digne de l'invention de Dante. Le kouros semble placé dans un contexte plutôt rassurant (à tel point qu'il peut se montrer curieux et observer des détails auxquels, en d'autres circonstances, il n'aurait pas prêté attention). Il est toutefois évident que, tout au long du voyage, il n'était pas en mesure de contrôler ce qui lui arrivait et qu'il n'avait aucune idée de ce qui pouvait lui arriver du fait de se trouver impliqué dans l'initiative des filles du Soleil. Ce n'est pas un hasard si la déesse, au moment de faire ses premières déclarations, éprouve le besoin d'introduire une précision rassurante : « nulle malfaisance, que ce soit bien clair!»

Est aussi soulignée une autre nouveauté : la multiplication des figures féminines (Parménide a pris soin de préciser l'identité sexuelle des cavales, des jeunes filles, de la déesse portière et de la déesse savante). En outre, alors que le kouros - unique mâle - est pour le moment réduit au rôle de spectateur des événements, l'initiative de l'action dramatique est confiée en totalité à un ensemble de présences féminines. En second lieu, l'une de ces présences féminines, celle dotée de la plus grande autorité, se révélera bientôt être la détentrice d'un savoir de tout premier ordre et de très grande ampleur, un savoir que le brillant jeune homme accepte sans réserve, sans commentaire et, semble-t-il, avec conviction. La même connotation de genre s'étend d'ailleurs aux autorités invoquées dans les frag-

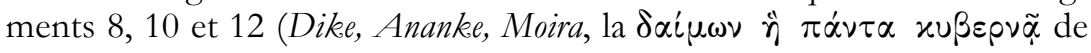


B12.4). Tout cela fait penser à une intentionnalité bien précise, même si elle reste pour nous mystérieuse.

Notons aussi les indices disposés en vue d'adoucir et d'atténuer la distance qui, objectivement, sépare le kouros de la déesse. À la différence du modèle hésiodique, où c'est le poète qui indique, demande et va presque jusqu'à prescrire l'objet du chant des muses, ici le kouros qui arrive en présence de la déesse se borne à écouter et n'essaye même pas de questionner. Il a suffisamment d'assurance pour penser qu'il ne doit pas être excessivement difficile pour lui (au moins pour lui) de suivre les discours de la déesse. Circonstance propre à rappeler la «définition» de sophos proposée par Pindare, illustre contemporain de Parménide, dans la

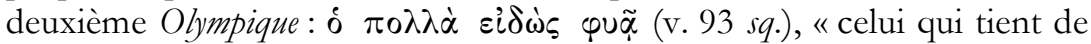
lui-même sa richesse intellectuelle ». Peut ainsi prendre forme, sans cependant être explicitée, une image élevée du kouros, interlocuteur doué et cultivé, dont sont reconnus les titres à recevoir les attentions de la déesse et la capacité de mettre à profit son enseignement, c'est-à-dire de le comprendre, l'apprécier et le faire sien.

Mauro Tulli a apporté une précision supplémentaire : à son avis, le kouros « parvient à la demeure de la déesse parce que son thymos en décide ainsi » et « la route lui appartient parce qu'elle pherei eidota phota, elle conduit l'homme qui possède un savoir ». Par conséquent pour le kouros du proème « le savoir ne vient pas à l'improviste, son investiture n'a pas lieu sans raison $»^{12}$. J'observe que ces deux détails sont dissonants par rapport aux nombreux indices qui montrent dans le kouros un spectateur inactif. L'explication consiste en ce qu'il aurait été aussi inopportun de faire du voyage le fruit d'une décision du kouros (ce n'est pas l'homme qui va à la déesse, c'est la déesse qui décide de se manifester à l'homme) que d'en faire un événement que le kouros se borne à subir (auquel cas on ne comprendrait pas pourquoi la déesse présuppose qu'il est en mesure d'apprendre). La voie médiane consiste, à ce qu'il semble, à introduire des indicateurs qui vont dans les deux sens, et en ce sens l'indication de Tulli est précieuse.

Venons-en aux processus d'identification. La condition du kouros qui ne sait pas bien ce qui lui arrive mais qui n'éprouve aucune crainte; qui, pendant le voyage, observe avec curiosité puis, une fois en présence de la déesse, ne peut que prêter la plus grande attention au discours qu'elle prononce (et ainsi, faut-il espérer, commencer à comprendre) - cette condition est une image transparente et créative de l'attitude que le poète veut encourager dans son auditoire. Face au poète qui chante son récit, le public se sent encouragé à se voir lui-même comme autant de kouroí, bien 
qu'auditeurs et lecteurs n'ignorent pas qu'en réalité ils sont passablement moins préparés que le poète narrateur, sans être pour autant complètement incapables de comprendre et d'apprendre. Mais il y a une autre chose que sait aussi chaque auditeur-lecteur : dans le mythos il est question de héros, de personnages de rêve, d'événements si exceptionnels qu'on ne peut imaginer qu'ils se répètent tels quels dans la vie courante; de ce fait, l'impréparation de l'auditoire ne fait pas problème : la logique du mythos est telle que, si n'arrivaient pas au personnage-narrateur des choses sortant de l'ordinaire, on n'aurait pas de raison de rester à l'écouter.

$\mathrm{Au}$ total, cette série de messages se traduit par une invitation catégorique à accompagner docilement le protagoniste dans les diverses opérations mentales que le poète-philosophe tient en réserve. L'auditoire est ainsi préparé à l'événement, au dévoilement, à ce qui pourra arriver, et à rien d'autre. Pour beaucoup de commentateurs de Parménide il a été et il est normal de s'appliquer à rechercher les indices de cohérence entre le proème et la philosophie proposée ensuite dans le premier logos de la déesse, mais il est permis de voir là une erreur de perspective, car le prologue n'expose rien de spécifique : il aurait été aussi approprié pour préparer le terrain à n'importe quel autre type d'événement imprévu. En effet, jusqu'au second hémistiche du vers 28 , on chercherait en vain des indices qui permettent de comprendre que le proème prépare l'auditoire au dévoilement d'une vérité particulière (et même d'une vérité primaire et d'un savoir d'une certaine façon secondaire) ${ }^{13}$. L'auditoire n'est pas en état de comprendre ce qui peut arriver, et même les premiers mots de la déesse n’apportent aucune lumière. La fonction préparatoire du proème est totalement non spécifique, dans la mesure où il n'a pas pour objectif de préfigurer le type d'enseignement qui sera délivré par la suite mais seulement de rendre l'auditoire attentif et réceptif.

\section{Le second prologue : les deux logoi et le changement des rôles}

Avec le vers $28 \mathrm{~b}$ la déesse passe, de toute évidence, à des préliminaires d'une tout autre nature, ce qui a pour effet de modifier le genre, la typologie, le système qui sous-tend ce qu'elle a à dire. Jusqu'à la moitié du vers 28 elle s'était bornée à rassurer le kouros au sujet des événements racontés auparavant (en soulignant, surtout au vers 24, l'aspect surhumain de son voyage hors normes). À partir de là, au contraire, elle paraît

13. On a vu parfois dans les Héliades qui se libèrent des kalyptrai (v. 10) la préfiguration de l'illumination qui libérera l'esprit du kouros des «brumes » de l'ignorance; dans les deux grands battants dont se composent les pylai (v. 13), la préfiguration des deux voies;

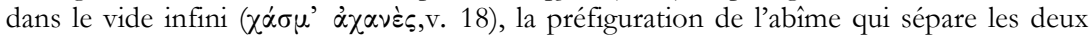
voies. J'avoue nourrir de sérieux doutes sur la validité de ces propositions d'exégèse. 
projetée en avant vers ce qu'elle s'apprête à dire, vers les enseignements qu'elle s'apprête à délivrer. C'est un changement si radical qu'il entraine l'oubli immédiat et définitif des événements qui viennent d'avoir lieu, lesquels, dans la suite, ne sont en effet plus évoqués et cessent littéralement d'exister pendant plusieurs centaines de vers, quitte à refaire surface tout au plus dans un bref épilogue ${ }^{14}$. Même si elle a bien rempli sa tâche propre, celle d'éveiller l'attention et de préparer le terrain, la narration préliminaire se voit ainsi, en substance, marquée d'insignifiance par rapport à ce qui tient proprement à cœur à la déesse et par conséquent au poète. L'objectif des vingt-sept premiers vers et demi était et ne pouvait être rien d'autre que l'identification, l'élaboration d'un horizon d'attente défini, la propension à porter un regard favorable sur le chanteur et sur ce qu'il s'apprête à proposer. Mais une fois tout cela accompli, la fonction ancillaire remplie par le voyage et par ses protagonistes n'a plus d'autre tâche ni d'autre domaine où s'exercer.

La confrontation avec la tradition épique pousse aussi à mettre en lumière des différences analogues. Alors que, dans les vingt-sept premiers vers et demi, on reconnait facilement une invention narrative relativement traditionnelle, à partir de la seconde moitié du vers 28 la tradition épique est brusquement mise de côté. À ce moment, en effet, la déesse, pour pouvoir faire comprendre au kouros qu'il s'agit maintenant d'en venir à savoir, qu'il s'agit de parler du monde réel et d'en pénétrer les secrets, ne peut faire autrement que laisser de côté la fiction narrative pour se concentrer sur quelque chose de radicalement différent de cette petite action dramatique. Elle tenait très fortement à faire savoir et à faire comprendre beaucoup de choses au kouros, et à cette fin elle l'a quasiment enlevé, mais arrive maintenant pour elle le moment d'abattre ses cartes, et en effet la déesse entreprend de présenter au kouros le savoir dont elle est détentrice, non seulement sans délai, mais aussi sans la moindre allusion au voyage initial. Les hodoi elles-mêmes du fragment 2 sont en effet complètement étrangères à l'hodos parcourue par le char : ce sont des « pistes » en un sens exclusivement mental et figuré.

Il survient cependant dans cette phase quelque chose de complètement inattendu. La déesse se met subitement à parler de deux savoirs : l'un, inébranlable et fixé à jamais, auquel devrait être attachée une totale crédibilité, et l'autre, infiniment plus précaire, même si, comme nous le verrons, il n'est pas dépourvu d'une valeur propre. L'annonce de deux types de savoir dotés d'un statut épistémologique profondément différent (chacun a son statut, avec des titres de créance différents) n'a pra-

14. En effet la logique de la situation semble impliquer que, à la fin du poème, la parole soit donnée de nouveau au kouros, peut-être seulement pour quelques vers. 
tiquement aucun précédent. On rappelle d'habitude un passage bien connu d'Hésiode mais, comme je l'ai déjà fait observer, l'analogie est plutôt faible puisque, chez Hésiode, nous avons une fiction narrative "fausse » que les muses opposent à un savoir "sérieux ", alors qu'ici on jette les bases de deux types de discours dont chacun prétend transmettre un savoir effectif, même si ces deux savoirs diffèrent au point de se révéler à peu de chose près incommensurables. Ce n'est donc pas un hasard si, à peine achevée, la fiction narrative cesse immédiatement de jouer un rôle et si, à partir du vers $28 \mathrm{~b}$, de nombreuses conventions de la poésie épique s'évanouissent rapidement. Ce qui prévaut maintenant, c'est l'exigence de donner un sens plausible à la coexistence annoncée de deux types de savoir très différents l'un de l'autre, et ensuite d'en venir à traiter du contenu de l'un et de l'autre. La déesse, en effet, se propose d'introduire le kouros non seulement à la connaissance d'une vérité eukykleos (B1.29) qui devrait être d'une crédibilité absolue et d'une dignité surhumaine, mais aussi à un savoir jugé éphémère, bon pour les mortels, mais qui, malgré ses limites évidentes (en particulier bien que sa crédibilité soit passablement modeste et qu'il soit peut-être frappé d'une instabilité intrinsèque), conserve cependant une valeur propre :

- il est, lui aussi, subjectivement nécessaire ;

- quoique limité, ce n'est pas un savoir quelconque mais, comme le confirme la déesse elle-même (B8.61), il est d'une plausibilité élevée ;

- surtout, il mérite d'être l'objet d'une exposition ample et détaillée de la part de la déesse; il acquiert (ou retrouve) par conséquent une dignité considérable, du fait de la décision de la déesse de délivrer aussi cet enseignement.

Une question s'impose donc: comment ce second type de savoir peut-il être «sans valeur, mais valable»? L'affirmation que le second savoir ne soutient pas la comparaison avec le premier, mais que ces choses-là aussi, il faut les connaître, le choix de ne pas se dérober à la tâche de faire part au kouros de ce savoir-là aussi, font penser que le poète a senti l'objection possible et qu'il s'est empressé de la neutraliser par avance. On notera que cette objection n'a rien à voir avec les «mystères » du voyage en char et du passage de la porte : elle met en cause uniquement la logique de l'enseignement à double face annoncé par la déesse ${ }^{15}$.

15. Ce que je veux dire, c'est que, si le premier logos est le vrai et si l'autre en est distinct, s'il n'en fait pas partie, il vient spontanément à l'esprit de concevoir le second comme faux, non digne de créance et par conséquent comme un hôte indésirable, un intrus. Ce n'est pas pour rien que la déesse elle-même le qualifie presque en ces termes et, à partir de Zeller, les commentateurs y ont vu "un simple complément, un simple accessoire, ou peut-être une croyance illusoire, voire une manière intrinsèquement fausse de 
En attendant nous apprenons que la déesse sait qu'elle sait, qu'elle sait même qu'elle possède deux types de savoir, qu'elle sait à quel point ils sont différents l'un de l'autre et que pourtant le savoir du second type a toujours une certaine valeur (elle n'ignore pas non plus à quoi il sert); qu'elle accepte de transmettre les deux mais qu'elle entend éviter tout risque d'équivoque ou de confusion et qu'elle est soucieuse non seulement de les distinguer soigneusement mais aussi de nous faire comprendre en quoi l'un diffère de l'autre. Notons, de plus, que nous pouvons et devons traiter le second logos aussi comme un savoir, dans la mesure où il se prête à faire l'objet d'un enseignement, à être appris et compris. En annonçant les deux types de savoir, la déesse entre dans le rôle du sophos et du didaskalos, cependant que le poète Parménide abandonne celui du chanteur qui cherche à enchanter et suggestionner son auditoire pour celui de l'intellectuel qui a appris et sait des choses retentissantes et qui est capable d'en rendre compte. Et de fait nous voyons son alter ego, le kouros, se faire l'élève très attentif de la déesse, appliqué à bien comprendre ce qui va lui être expliqué petit à petit. Le cadre épique commence ainsi à se remplir de contenus qui lui sont étrangers, donc à se réduire à n'être qu'un cadre rassurant, alors que ce qui désormais définit la situation, ce sont les idées nouvelles, les exigences intellectuelles inédites, le problème, les attentes, les priorités ${ }^{16}$.

Indirectement, l'horizon d'attente change aussi : les auditeurs/lecteurs aussi cessent de se représenter eux-mêmes comme spectateurs et, d'une certaine façon, ils deviennent des élèves, des personnes qui se mesurent à des discours difficiles, qui s'appliquent à bien comprendre (et donc, comme nous le verrons, à mûrir leurs jugements) et qui surtout se sentent gratifiés de pouvoir accéder à des idées neuves. De même pour les processus d'identification: les auditeurs/lecteurs comprennent - nous comprenons - qu'en s'adressant au kouros c'est à eux - à nous - que s'adresse la déesse, parce que, en réalité, c'est nous qui devons (et voulons) vivre l'expérience gratifiante d'accéder au savoir, au savoir de choses proprement inouies. Et si c'est nous qui sommes les véritables destinataires, alors la fiction narrative nous assimile au poète lui-même qui écoute avec la plus grande attention: nous aussi, nous nous sentons

dire comment sont les choses » (Rossetti 2008a, p. 11). "Aussi longtemps que Parménide et la déesse tiennent le mot "être" pour un prédicat univoque qui exprime la nature véritable de son sujet», "les opinions des mortels demeurent désespérément invalides » (Granger 2002, p. 115 et 102). Voir aussi sur ce thème Robbiano 2006, p. 117 sq. et Thanassas 2007, p. 19.

16. Le proème est aux deux logoi « comme les cloches d'une église qui annoncent une célébration liturgique mais par elles-mêmes ne disent rien, qui doivent seulement annoncer et attirer » (Luis Fallas, dans un livre à paraître prochainement). 
invités à écouter avec toute la concentration nécessaire. Nous en venons ainsi à nous voir nous-mêmes, au moins tendanciellement, comme des gens capables de recevoir et de comprendre le double enseignement. Ainsi peut prendre forme une captatio benevolentiae de haut vol : nous sommes nous aussi jugés dignes d'accéder à une vérité supérieure et, en supplément, à un savoir qui est davantage à l'échelle humaine. Nous commençons la lecture en spectateurs, pour un peu nous nous sentons « en tiers » vis-à-vis des personnages du récit, mais nous en venons rapidement à deviner que, grâce au degré élevé d'élaboration de son initiative communicationnelle, à partir de maintenant ce n'est que sous l'horizon de la fiction narrative que la déesse parlera au poète. La conséquence, c'est que la déesse elle-même disparaît : sa place est prise, au moins tendanciellement, par le Parménide réel qui se prépare à nous délivrer, l'un après l'autre, les deux groupes d'enseignements annoncés. Entre déesse, kouros, Parménide en tant qu'intellectuel historiquement situé et auditeur potentiel s'effectue un glissement des rôles novateur en termes de création et de nature à établir une césure on ne peut plus nette entre le prologue narratif et les enseignements qui s'annoncent, un glissement indiscutable mais qui, à en juger par les interprétations dominantes, a tendance à passer inaperçu, comme s'il s'agissait de la chose la plus naturelle du monde.

Observons enfin que, si le prologue au sens strict est métadiscursif dans le sens où il dessine le cadre de l'ample initiative communicationnelle qui va suivre, les premiers mots de la déesse sont métadiscursifs en un sens plus éminent, parce qu'ils parlent des discours que la déesse va prononcer, des caractéristiques qui les différencient et de l'opportunité de présenter non pas seulement le premier mais aussi, malgré ses limites, le second type de savoir.

\section{Le premier logos. La fonction assignée aux fragments 2-7}

Passons donc au logos véridique. Il est recouvert, pour ainsi dire, de trois enveloppes protectrices : il y a d'abord le décodage d'une situation relationnelle complexe (le destinataire doit comprendre que l'événement se présente comme un enlèvement forcé mais, en compensation, plaisant et, à sa façon, raisonnable), puis l'annonce des deux logoi (il faut arriver à l'idée que seront donnés deux discours si différents qu'ils se révéleront étrangers l'un à l'autre) ; et maintenant, une fois annoncé le commencement du premier logos, vient le tour d'un troisième type de préliminaires qui, nous allons le voir, servent directement au déploiement de cet enseignement lui-même : ce sont les notions nécessaires pour comprendre, et ainsi entrer graduellement dans l'univers mental du premier logos et se 
familiariser avec lui (ce qui fait qu'il est parfaitement correct d'évoquer à ce sujet les notions de dévoilement et de révélation) ${ }^{17}$.

On sait qu'il y a de fortes raisons d'attribuer aux fragments 2-7 un rôle préparatoire par rapport au développement concentré dans les quarante-neuf premiers vers du fragment 8. Ce qui fait penser à un groupe de considérations préliminaires, c'est, en particulier, l'impossibilité matérielle de supposer que ces six fragments puissent trouver place après le développement principal qui nous est parvenu ${ }^{18}$. Cela veut dire que Parménide a certainement concentré toutes ces considérations préliminaires dans une seule section du poème, celle qui précédait l'excursus ontologique du fragment 8 . Il est d'ailleurs évident que ces considérations jettent les bases d'un savoir sans s'appliquer à sa construction explicite, et elles se caractérisent par l'homogénéité de leur rôle. De fait, dans les fragments 2-7 la déesse aborde des thèmes complémentaires, dont on devine qu'ils sont distincts l'un de l'autre: les hodoi, la relation êtrepensée, dire-penser, dire-comprendre, le noos, la notion même d'être, le point de départ, la soi-disant troisième voie... Chacun de ces thèmes connaît un développement propre, et tous ensemble servent à explorer et à marquer le territoire en introduisant, à titre de préliminaires, certaines alternatives essentielles.

En particulier, l'identification de la voie sur laquelle il n'y a pas lieu de s'aventurer (fragment 2) permet de caractériser chacune des deux et de donner une idée des raisons pour lesquelles l'une est bonne et l'autre non. La notion qui domine ici est celle de pensabilité, suggérée par voñ$\sigma \alpha \iota$ au vers 2 et reprise au vers 7 par $\gamma$ voins. On arrive ainsi à un premier résultat: diverses hypothèses ont été prises en considération et on a vu que, après une première sélection, deux seulement subsistent et méritent

17. Je passe sur la formule de transition qui crée une discontinuité supplémentaire, au point de vue fonctionnel, entre le premier vers du fragment 2 ( « eh bien, je te le dis maintenant, et toi, veille à être bien attentif pendant que tu écoutes ce que je dis ») et les vers suivants, parce qu'il s'agit d'une donnée purement formelle.

18. Il pourrait y avoir un doute concernant le fragment 4 puisque, théoriquement, le v. 3 ("ni entièrement dispersé dans chaque partie du cosmos », comme traduit Cerri 1999) pourrait aussi évoquer quelques éléments supplémentaires du second logos, et que le

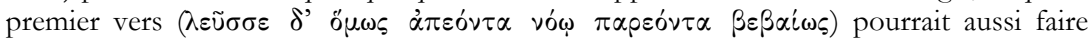
penser à une sorte de péroraison finale. Ce n'est donc pas un hasard si nous lisons dans Ramírez Vidal 2005, p. 100 : «Le Poème se serait conclu par un épilogue (fr. 19 et 4), qui était peut-être une exhortation à contempler la vérité (fr. 4). » On peut soutenir au

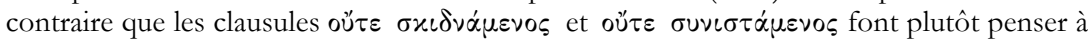
l'énoncé de directives pour la pensée, donc précisément aux considérations préliminaires auxquelles il convient d'assigner les fragments $2-3$ et 5-7. 
d'être prises en considération ${ }^{19}$; on a compris, de plus, que l'une des deux routes peut être parcourue, tandis que l'autre ne peut absolument pas l'être. Il devient clair, de cette façon, que Parménide (ou, si l'on préfère, sa déesse) est en train de présenter les résultats d'une enquête préliminaire, le savoir qui a pris forme en procédant par élimination grâce au

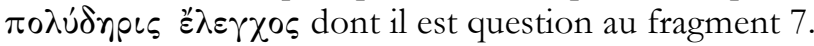

Un message analogue est exprimé par le fragment 5 , qui évoque de nombreuses tentatives antérieures de développer et d'accréditer le même thème, et par les fragments 6 et 7, qui font entendre un sentiment d'aversion pour qui se refuse à accepter l'enseignement du poète-philosophe. Ces rappels d'une recherche et d'un enseignement qui se sont prolongés dans le temps évoquent un contexte de discussions entre sophoi, le rejet, pour l'essentiel, de points de vue en vogue, et une longue recherche qui, à en juger par ce que nous lisons, devrait avoir eu lieu (et s'être conclue) avant que Parménide ait mis la main au poème. C'est pourquoi les considérations exposées dans les fragments 2-7 sont préliminaires et propédeutiques ${ }^{20}$ : non seulement elles servent à chasser tant de préjugés, mais elles présentent aussi les fruits d'une recherche et d'une réflexion qui a déjà eu lieu auparavant. Une telle référence au processus de décantation qui a rendu possible l'élaboration et la fixation d'un enseignement déterminé est extrêmement rare. Qu'une telle donnée témoigne en faveur d'une très nette césure entre le proème et les contenus doctrinaux n'est pas moins digne de remarque.

Ces préliminaires ne se bornent pas à nous faire savoir qu'«ici la route est facile, alors que là elle est pleine d'embûches » (donc à nous rassurer en même temps qu'à nous mettre en garde), mais ils définissent un critère et en motivent l'adoption, de façon que l'auditoire apprenne à s'orienter et à s'autoréguler, jusqu'à former sa propre vision des choses. Ce que Parménide semble s'appliquer à nous proposer dans cette section ne se présente donc pas comme un assemblage d'informations, mais comme une structure de type métacognitif ${ }^{21}$ qui pousse à penser, et à penser d'une façon bien précise ( $c f$. fr. 6 et 7). Une telle attitude suppose que l'auteur se propose non seulement d'enseigner (et éventuellement de

19. On devine qu'il y a eu un processus de sélection préliminaire, une réflexion sur les alternatives possibles. À la vérité, si l'une des alternatives consiste dans l'idée d'un être entièrement dégagé de toute relation, l'alternative logique serait celle d'un être plongé dans le système de relations, éventualité expressément rejetée dans le fragment 6. Mais, pour affirmer un être complètement dégagé du réseau des relations, il est beaucoup plus commode de lui opposer un non-être semblablement dégagé, parce que cela rend plus facile d'affirmer la notion d'être qui tient à cœur à Parménide.

20. Cerri 1999 parle de «prémisses méthodologiques ».

21. Pour la notion de métacognitivité, je me permets de renvoyer à Rossetti 2008b. 
proposer certaines idées de manière à les inculquer et qu'elles soient intériorisées : fr. 5), mais aussi de faire en sorte que l'auditoire parvienne graduellement à partager le point de vue du locuteur, en développant sa disposition à raisonner " juste », donc une façon de regarder, d'observer et de reconnaitre, un critère que l'auditeur devrait pouvoir comprendre, approuver et progressivement intérioriser, jusqu'à partager absolument les mêmes critères (et les mêmes jugements) que le locuteur. Nous pouvons donc dire qu'est ici établi un processus d'acculturation sous l'effet duquel l'auditoire virtuel en vient à partager les options de base qui animent cet enseignement, en fixant fermement dans l'esprit quelques étapes avant même d'aborder le sujet et de développer le discours.

L'énergie mise à porter l'auditoire à partager non seulement tel ou tel point, mais toute une façon de penser, constitue donc quelque chose de plus que la simple élaboration d'un horizon d'attente ou que le simple exercice de la fonction didactique. En fait, Parménide ne se borne pas à nous donner des assurances sur le fait que la voie où s'engage le poètedidaskalos est la bonne : il vise aussi à instituer un organigramme complet de présupposés fondamentaux, susceptibles d'être partagés et donc aptes à former des personnes (toute une génération de nouveaux intellectuels ?) qui se tiennent fermement à certaines idées essentielles. Paraît donc un heureux choix la notion de "philosophie transformative " récemment introduite par Robbiano (2006), car le poème montre qu'il a pour objectif de transformer son auditeur attentif en un intellectuel capable de mettre au point un critère et de se reconnaitre dans une nouvelle façon de «lire » la réalité. Il ne serait pas moins exact de parler de stratégie macro-rhétorique ${ }^{22}$, dans la mesure où ici une série complète et coordonnée d'étapes est conçue dans le but d'acclimater l'auditoire à une façon de penser très précise, de créer un terrain d'entente commun et des conditions favorables à l'acceptation du noyau doctrinal primaire qui va suivre. L'aboutissement de cette stratégie est la fixation de règles nouvelles et rigides pour l'emploi de notions telles que estin et ouk estin, alors que les gens sont habitués à passer de l'une à l'autre et à les combiner selon des modèles codifiés (p. ex. «cette fleur n'est pas jaune, elle est rouge », " aujourd'hui tu as eu une petite sœur, pas un petit frère »), donc sans précautions particulières. L'idée qu'inculque le poème, c'est que, s'agissant de notions incompatibles entre elles, il faut adopter des critères particulièrement rigoureux pour les employer ${ }^{23}$.

22. Cf. Rossetti 1994.

23. Plus précisément, sont élaborées ici les notions d'un être et d'un non-être affranchis du système de relations qui prend forme grâce aux énoncés affirmatifs et négatifs (p. ex. «A est $p$ mais n'est pas $q$ »; « parce que justement il est $p$, on peut comprendre que 
Il vaut cependant la peine de relever aussi l'affinité notable entre cet ensemble de considérations introductives et toute la série de préliminaires (définitions, postulats et notions communes) qui occupent le début des Éléments d'Euclide ${ }^{24}$. En effet, nous n'avons pas de difficulté à comprendre que ces précisions sont ordonnées (c'est-à-dire nécessaires, mais aussi aptes à fournir un support efficace) au parcours démonstratif qui va suivre. À son tour la fonction uniforme de ces considérations induit à penser que Parménide les a traitées précisément comme des prémisses logiques : même si, évidemment, il ne pouvait faire état de cette notion, il semble avoir saisi la spécificité de leur fonction logico-argumentative en tant que conditions préliminaires. La capacité de traiter les prémisses comme des prémisses constitue donc une des nouveautés qui caractérisent le poème.

Il faut aussi relever l'ampleur de cette phase spécifique de préparation de l'auditoire: ce que nous pouvons encore lire, c'est un ensemble de trente vers, marqué d'incontestables lacunes ; il est par conséquent vraisemblable que sa longueur entière était égale ou supérieure aux quaranteneuf vers dans lesquels se déploie la section la plus spécifiquement ontologique du poème.

\section{Le noyau central du premier logos. Les instruments de la dispute doctrinale}

Ce qui rend nécessaire une infrastructure argumentative aussi élaborée, c'est de toute évidence le degré élevé d'abstraction de la théorie de l'être et sa propriété d'aller à l'encontre des évidences de l'expérience quotidienne. En fait, privé du soutien des prémisses dont il a été question ci-dessus, l'exposé principal ne pourrait aucunement prendre son essor, parce qu'il s'exposerait à des objections capitales. Inversement, l'ensemble de préliminaires que constituent les fragments 2-7 sert d'excellente façon à interdire le recours aux usages linguistiques ordinaires, qui seraient affectés d'une sorte de péché mortel dans le sens précisé plus haut (notes 19 et 23).

Il est compréhensible que le fragment 8 ait retenu l'intérêt d'un vaste cercle de spécialistes : il présente une sous-unité textuelle indiscutablement complète, quarante-neuf vers qui introduisent, développent et con-

A ne soit pas $q »)$. Alors que ce système de relations rend les deux termes complémentaires, limitrophes et donc interdépendants, le choix de s'en passer permet d'absolutiser les deux termes et d'arriver à ce que l'un soit à l'autre exactement comme le tout au rien (et à un rien qui ne soit même pas l'espace dans lequel trouve place le tout).

24. Un premier pont a été opportunément jeté entre Parménide et Euclide dans Marcacci 2009, p. 69-72 et 85. 
cluent un parcours argumentatif complet, pour continuer ensuite avec une formule de transition qui clôt un discours et en ouvre un autre. Nous savons donc avec certitude que, après les préliminaires analysés ci-dessus, le moment était venu pour la déesse de Parménide d'en tirer la conclu-

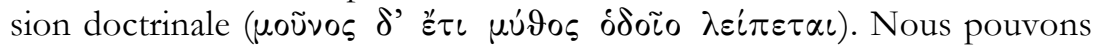
voir clairement que cette conclusion - authentique climax de tout le premier logos - se divise en l'affirmation péremptoire " que est », le passage en revue des cinq (ou six) semata relatifs aux caractéristiques de l'eon et, tout de suite après, le commencement d'un superbe itinéraire démonstratif. Les semata ont le statut de demonstranda (l'être est ageneton, anoletbron, oulomeles, atremes et ateleston) et l'exposé se poursuit par un développement qui reflète la succession des demonstranda, s'arrêtant avec l'emphase convenable sur l'alternative fondamentale, présentant un emploi récurrent et inhabituel de la conjonction gar au double sens de because (" $p$ étant donné que $q$ ») et de then (" si tout cela est vrai, alors il est vrai aussi que $p »$ ) et faisant même place à des expressions qui se rapprochent de façon significative du classique quod erat demonstrandum des mathématiciens ${ }^{25}$. Les expressions qui équivalent au $Q E D$ sont les suivantes :

Alors (oü $\tau \omega \varsigma$ ) il est nécessaire qu'il soit complètement ou qu'il ne soit pas du tout

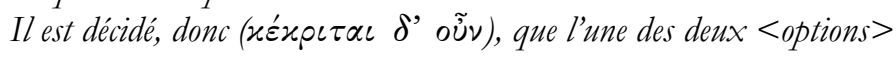

... tandis que l'autre...

B8.11

Et ainsi $\left(\tau \omega^{26}{ }^{26}\right)$ la naissance est éliminée, à l'égal de la mort obscure

B8.16-18

En vertu de quoi $(\tau \tilde{\omega})$ il est tout entier continu,

en effet l'être s'attache à l'être

B8.21

En vertu de quoi $(\tau \tilde{\omega})$ seront des noms toutes ces choses...

B8.25

Non moins significativement, après chacun de ces énoncés formant la conclusion de chaque démonstration apparaissent des formules de transition, indiscutablement paratactiques, qui, de toute évidence, servent à ouvrir la voie à un nouvel itinéraire démonstratif :

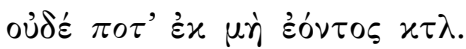

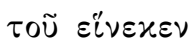

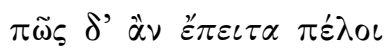

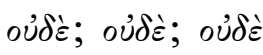

$\alpha \dot{u} \tau \dot{\alpha} \rho \dot{\alpha} x i \nu \eta \tau \sigma \nu \mu \varepsilon \gamma \dot{\alpha} \lambda \omega \nu \quad x \tau \lambda$.

25. On trouvera quelques anticipations importantes de ces remarques dans Marcacci 2009.

26. Bollack 2006, p. 153, traduit même: «Dans cette logique, la naissance est éteinte...». C'est un choix qui a sa logique. 


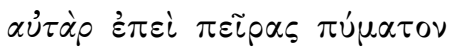

De fait, le cadre poétique n'empêche pas de comprendre que l'exposé sous-jacent à une grande partie du fragment 8 est régi par une infrastructure logico-argumentative très élaborée, très mûre, comme si l'auteur avait eu auparavant de nombreuses occasions d'affiner son propos et fût ainsi parvenu à se représenter nettement les passages, les étapes, les articulations de son raisonnement. On peut rappeler à ce sujet le $\pi 0-$

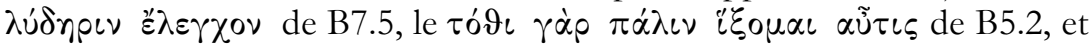
le $\alpha \tau \rho \varepsilon \mu \dot{\varepsilon} \varsigma \tilde{\eta} \tau o \rho$ de B1.29, qui évoquent une sorte de longue bataille, une bataille que Parménide semble avoir eu à soutenir pour vaincre des résistances tenaces avant même de commencer la rédaction de son poème. La référence à la krisis en $\mathrm{B} 8.15$ évoque aussi des résistances et une procédure adoptée pour sortir de l'impasse et arriver à une décision (l'alternative suppose que, si l'une des deux possibilités est exclue, par là même l'autre est affirmée).

Ce thème des résistances et des critiques adressées à la théorie principale de Parménide refait surface, comme on le sait, en rapport avec les buts poursuivis par Zénon au moyen de ses paradoxes, et on peut même trouver séduisante l'idée que la théorie ait été attaquée avant même de prendre forme et d'être rendue publique dans le poème ${ }^{27}$. Indépendamment de cette éventualité, les nombreux indices recueillis jusqu'ici nous font savoir que Parménide a prévu et intériorisé de telles résistances et qu'il en a reconnu la force. Il n'a d'ailleurs pas un grand effort à faire pour avoir à s'attendre à des résistances, car sa théorie dessine une situation radicalement différente de celle dans laquelle nous nous trouvons tous immergés (décrochage de l'être du système des relations : note 23) et elle prétend en outre que les choses doivent être nécessairement de la façon qu'elle indique. Incombait nécessairement à Parménide, par conséquent, la charge de démontrer que les choses ne peuvent être d'aucune autre façon, c'est-à-dire d'expliquer pourquoi la réalité la plus vraie et la plus originaire ne peut être née, finir ni comporter de différences. En d'autres termes, pour quiconque venait à connaître cette théorie, il devait être absolument normal d'intervenir par un «mais pourquoi donc? ? et de manifester sa perplexité. Parménide ne pouvait donc pas ne pas s'attendre à ce type de questions, d'objections et finalement de résistances. L'idée que sa théorie ait pris forme justement grâce à un $\pi \circ \lambda u ́ \delta \eta p \iota \varsigma$ है่ $\varepsilon \gamma \chi 0 \varsigma$, à un rejet de l'opinion courante, formulé et argumenté maintes fois et de maintes façons, affiné avec le temps avant que prenne forme le poème - cette idée n'est pas une conjecture 
fantaisiste. Nous pouvons conclure, par conséquent, que, ayant à se mesurer avec de prévisibles et tenaces résistances, Parménide a probablement fini (a) par pleinement contrôler ses propres stratégies argumentatives et communicationnelles, (b) par élaborer, en fonction du premier logos, un parcours démonstratif paradigmatique, susceptible de manifester un niveau particulièrement élevé de maitrise de son argumentation, et (c) par mettre au point des stratégies sophistiquées de protection de sa théorie, des défenses non éphémères, clairement conçues et vraisemblablement efficaces. De fait, la qualité des stratégies que Parménide a établies et mises au point est particulièrement élevée, trop élevée pour ne pas penser qu'il se soit longuement employé à affûter ses $\operatorname{armes}^{28}$.

Les stratégies adoptées dans la construction de la théorie ne se limitent pas aux moyens décrits ici, et il serait possible d'en pousser encore plus loin l'étude. Cependant l'importance objective de l'architecture dont nous avons repéré les lignes porteuses incite à mettre en lumière non pas tant des détails supplémentaires que l'ars combinatoria qui est ici à l'œuvre, autrement dit la façon dont des «éléments architectoniques» sont combinés entre eux pour donner finalement cette combinaison particulière et aucune autre. De fait, les différentes parties sont adaptées les unes aux autres de façon impressionnante : une préparation à distance (le proème, avec intervention de diverses figures supra-humaines, puis l'annonce explicite d'un logos irrésistible et inattaquable), un ensemble structuré de considérations préliminaires (ou préjudicielles) abordées une par une, puis l'énonciation de tout un groupe de demonstranda, puis la démonstration du bien-fondé de chaque demonstrandum (avec, chaque fois, la réussite de la démonstration soulignée par un $Q E D)$, puis la configuration du résultat ( l'être est donc fait ainsi ; nous pouvons nous le représenter comme une sphère... »); enfin la formule de transition par laquelle le poète annonce que le pistos logos est terminé. Comme on le voit, nous sommes en présence d'un projet où peu de place est laissée au hasard. On peut donc légitimement supposer que Parménide a longuement médité la composition de son poème, réfléchi à ses embûches et à ses points forts, aux façons d'en traiter, pour finalement choisir un projet architectonique déterminé et s'y fier.

Nous nous en rendrons mieux compte si nous examinons les indices de complémentarité entre les considérations préliminaires que nous con-

28. Nous trouvons un motif d'étudier les stratégies de construction du poème précisément dans le fait que nous sommes en présence d'un enseignement beaucoup moins improvisé, beaucoup plus «construit » et "étudié», beaucoup plus conscient que beaucoup d'autres. 
naissons, et donc le degré de congruence entre la série des demonstranda et les démonstrations effectivement offertes au long du fragment 8 .

Pour ce qui est des considérations préliminaires (fr. 2-7), il est facile d'en dégager l'idée centrale (fr. 2, 4.2-4 et 6.1-2) qui consiste dans l'affirmation que la relation entre être et non-être n'est pas assimilable à celle entre la lumière et l'obscurité (avec les innombrables variations de l'ombre et de la pénombre) ou entre le plein et le vide (avec la possibilité que le plein ne soit pas complètement plein ni le vide totalement vide), parce qu'il n'y a pas de sens à dire qu'un être est mais, à certains points de vue, peut aussi ne pas être, et surtout il n’y a pas de sens à traiter le non-être comme quelque chose (plutôt que le pur néant). L'introduction d'une notion précise d'être (et de non-être) constitue de toute évidence le pilier fondamental de tout le premier logos, au sens où un accord sans réserve sur ce point précis constitue la condition indispensable pour parer à l'incompréhension quand le poète en viendra à de nouvelles inférences, cruciales, à propos des caractéristiques de l'être. Qualifier d'insensé quiconque ne comprend pas cette sorte d'axiome fondamental (fr. 6.4-9 et 7) constitue un complément ou une confirmation de l'impératif d'avoir à approuver et partager cet axiome. On peut reconnaittre un second complément dans les considérations sur dire-penser et penser-être (fr. 3 et 6.1, avec une reprise en 8.8 et 8.34-40), expressions dans lesquelles je ne crois pas qu'on puisse identifier une doctrine spécifique, mais plus simplement une manière de souligner combien sont justes et naturelles les réflexions qui induisent à pousser à l'extrême l'écart entre être et néant. En revanche, c'est en un autre sens que le fragment 4.1 est préliminaire : il invite à considérer la réalité dans son ensemble et/ou sous son aspect le plus caractéristique et le plus originaire. À son tour, le fragment 5 exhorte à aborder l'exposé à partir de différents points de vue, de manière à parvenir à un plein assentiment. Ce que met en relief cette construction, donc, c'est d'un côté la fixation sans équivoque d'un enseignement fondamental, et de l'autre l'extrême importance attribuée à cet enseignement, de façon que l'auditoire accepte de mobiliser toute son énergie intellectuelle dans le but d'arriver à comprendre les raisons pour lesquelles est affirmée une idée particulière de l'être (et du non-être). Ainsi prend forme, au cours même des considérations introductives, une hiérarchie fonctionnelle qui désigne ce que le poète tient pour crucial et ce qui, au contraire, doit servir de support et d'appui aux thèses les plus importantes.

Passant maintenant à l'examen du fragment 8.1-49, je propose d'évaluer l'adéquation entre l'annonce de chaque demonstrandum, la façon dont il est démontré et la déclaration finale correspondante. 


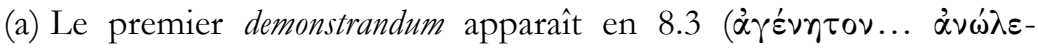
Эpov) ; la démonstration correspondante est donnée une première fois en 8.6-10 et, avant d'être reprise en 8.21, elle se conclut en 8.11 par la déclaration qu'elle a été menée à bonne fin. La déclaration du vers 11 revêt une signification très particulière du fait qu'elle n'a aucun précédent et qu'elle constitue donc une nouveauté : c'est la première formulation qui nous soit connue de ce QED qui dans la suite est devenu topique, mais qui n'est documenté qu'à partir des Éléments d'Euclide, c'est-à-dire un bon siècle et demi plus tard. Quant au fond, ce qui frappe, c'est l'affirmation que l'être ne peut naître du néant (i) parce que le néant, puisqu'il n'est rien, n'est pas non plus en mesure d'expliquer la naissance ou l'apparition de l'être, (ii) parce que, dans cette hypothèse, ferait défaut la cause suffisante, le fait nouveau à la suite duquel aurait pu se produire la génération de l'être à partir (et par l'action) du néant. Parménide fait clairement comprendre qu'il adopte une acception non empirique de l'être, qu'il parle d'un être pur et originaire, d'une situation archétypique où, à côté de l'être, il n'y a pas de place pour le néant. Passage crucial, non seulement parce que l'idée est ensuite reprise de façon répétée, mais parce que, comparée aux considérations développées dans les fragments 2-7, c'est une idée nouvelle qui est introduite ici : si le néant est vraiment néant, alors l'être ne peut connaître ni naissance ni mort (ni, selon l'argumentation qui va suivre, un autre-que-soi, ni divisions ou différenciations en son propre sein, ni transformations). On notera l'absence d'une précision fondamentale, qui a induit en tentation d'innombrables commentateurs, mais qui peut être postulée par inférence : la référence à la condition originaire du monde, détail qui, de fait, reste implicite ${ }^{29}$.

(b) L'argumentation donnée à l'appui du deuxième demonstrandum

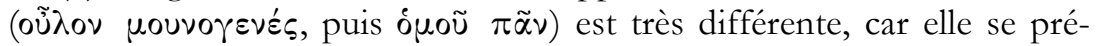
sente comme un simple corollaire de l'argumentation précédente, au point de se réduire au seul énoncé de la thèse. Ce qui complique un peu les choses, c'est que sa formulation ne correspond au demonstrandum que conceptuellement. Pour démontrer que l'être constitue le tout et qu'il revêt la forme d'un bloc compact, est affirmée en effet l'impossibilité que quelque chose d'autre naisse du néant et vienne s'ajouter à lui. La relation entre les deux déclarations est de type contrefactuel, en ce sens que, si à côté de l'être apparaissait quelque chose qui en resterait distinct, l'être ne constituerait plus le tout, ni un bloc compact. La nouvelle déclaration offre l'avantage supplémentaire d'être strictement complémentaire par rapport à la précédente (l'être ne peut pas sortir du néant $\sim$ rien d'autre

29. Il faut noter cependant la correspondance entre cette démonstration et le premier trait indiqué en 8.5-6: ขบ̃v. 
ne peut sortir du néant et s'ajouter à lui). Selon Parménide, donc, le deuxième demonstrandum ne serait sans fondement (non démontré) que si l'on admettait que de nouvelles entités peuvent gignesthai et rester distinctes de l'être.

(c) En 8.12-18 apparaît l'évocation des interdits de Dikè, accompagnée de l'indication explicite de la krisis : le point critique, la question sur laquelle porte la décision à prendre. Dans ce passage (8.15-18) nous voyons Parménide s'enferrer dans une situation en soi tautologique; en effet il se borne à répéter ce qui a été d'emblée proclamé dans le fragment 2, c'est-à-dire peut-être quarante à cinquante vers avant d'en arriver à la nouvelle formulation. Surprend aussi, dans ces vers, l'accumulation notable de termes non spécifiques (p. ex. pedesin) et d'hypostases comme Dikè et Anankè ; cette accumulation a pour effet d'estomper le caractère novateur de la détermination explicite - de grande valeur analytique - du point crucial. Il est possible que les vers 12-18 servent à démontrer le

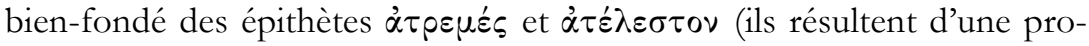
cédure par élimination), étant entendu que dans ce cas la correspondance avec le troisième demonstrandum reste vague ${ }^{30}$.

(d) En 8.19-21 commence le parcours démonstratif correspondant à 8.5 («il n'était pas, il ne sera pas »). La thèse comporte un rejet de l'anthropomorphisme, au sens où ni le verbe pelo ni le verbe gignomai ne peuvent s'appliquer à l'être, parce qu'ils se réfèrent à une acception complètement différente de l'être : alors que dans l'acception courante de ces termes on existe et on naît (c'est-à-dire qu'on se trouve et qu'on prend place en plus de ce qu'il y a déjà), il est répété ici que, à côté de ou face à l'être, il n'y a pas même de place pour le néant (parce que, comme il a été établi dans les prémisses, le néant n'est pas quelque chose). Peut ainsi prendre forme et être rendu crédible, en 8.20, un double énoncé ouvertement contraire à la perception commune de la réalité (et non moins paradoxal que certains énoncés d'Héraclite) : s'il était né, il ne serait pas ; s'il devait naitre dans l'avenir, il ne serait pas. Dans le contexte, la phrase ne scandalise pas, parce qu'il y a déjà eu une éducation collective à l'utilisation de catégories mentales réformées sur la base de l'asymétrie totale entre être et non-être, mais on notera qu'une justification n'est pas même évoquée. En fait, il manque un maillon de la chaîne déductive, semble-til. Il est d'ores et déjà admis, à juste titre, que la naissance ne peut avoir lieu, dans la mesure où elle comporterait le passage du non-être à l'être et donc l'attribution d'un rôle générateur au non-être ; la mort, de même, comporterait l'attribution au non-être de la capacité de "faire mourir » l'être. Dans les deux cas, au pur non-être serait ainsi attribué un pouvoir

30. Cf. McKirahan 2008, p. 195 sq. 
immense, ce qui est proprement $\ddot{\alpha} \pi v \sigma \tau o v$. À partir de ces prémisses, nous comprenons que les notions de mort et de naissance ne sont introduites ici que pour être soumises à un mémorable renversement: à la lumière de la théorie, des expériences universelles et bien connues comme naitre et mourir deviennent complètement impensables, légendaires, fantaisistes, absurdes. La théorie s'affirme contre des expériences universelles qui sont condamnées parce que incompatibles avec la théorie. Cet extraordinaire passage constitue vraisemblablement la prémisse sur la base de laquelle les intellectuels influencés par Parménide (pas seulement Anaxagore) ressentiront bientôt l'exigence de sauver les phénomènes, étant entendu qu'il ne s'agit pas de nier le fait de la naissance et de la mort en général, mais de nier que naissance et mort puissent concerner l'être dont il est question.

(e) Les vers 8.22-24 introduisent aussi des notions jamais encore rencontrées (et vraiment cruciales): d'un côté celles d'indivisibilité et de compacité, de l'autre celles d'homogénéité et de plénitude (É $\sigma \tau \iota \nu$ ó $\mu$ õ

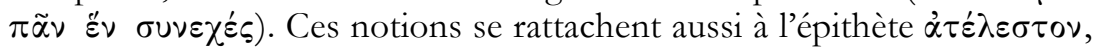
à condition de comprendre - comme il le faut - que la présence de divisions (fractures, différences, discontinuités) au sein de cet être-arche qui n'admet même pas auprès de lui le néant serait un indice d'imperfection et d'inachèvement et, surtout, serait inconcevable.

(f) Nous avons ensuite le corollaire brièvement évoqué en 8.25 , destiné à rendre explicite l'idée de compacité et d'achèvement de l'être, avec

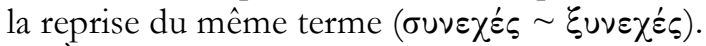

À une seule exception près, partielle, ces six sections correspondent donc bien à l'objectif d'argumenter sobrement, mais point par point, les demonstranda énumérés au début. Cela souligne l'importance des nombreuses déclarations finales assimilables au QED des mathématiciens, et en même temps cela confirme que Parménide a composé consciemment cette partie de son poème et d'autres : il sait qu'il sait, il sait ce qu'il veut démontrer, il est conscient des difficultés qui se présentent et il a des idées précises sur la façon de les surmonter. Dans les vingt-cinq vers que nous venons d'examiner, le poète-philosophe donne des preuves et fait remarquer qu'il a donné des preuves grâce auxquelles sa thèse peut être tenue pour confirmée et accréditée. Certes, il ne peut avoir idée des formalisations logico-mathématiques (évidentes pour nous), mais il ne se laisse pas conditionner par la forme poétique et il se montre déterminé à soutenir que ses vers laissent entrevoir la discipline intellectuelle qui préside au schéma démonstratif qu'il a mis au point.

Un autre point à observer concerne l'exhaustivité des prémisses. L'examen des demonstranda permet de constater qu'il en manque au moins une. Comme on l'a observé dans la section (d) ci-dessus, il semble man- 
quer un maillon de la chaîne déductive. Parménide ne nous dit pas pour quelle raison précise la naissance comporterait le passage du non-être à l'être (donc l'attribution au non-être d'une fonction génératrice qui ne lui convient pas), ni que la mort comporterait l'attribution au non-être de la capacité supplémentaire de «faire mourir » l'être ; il ne nous dit pas que, si l'on raisonne de cette façon, le pur néant se verrait attribuer un pouvoir immense, ce qui est proprement $\alpha \pi v \sigma \tau o \nu$. Mais il devait avoir clarifié quelque chose de ce genre au cours du poème. S'il l'a fait, ce sera certainement dans le cadre des prémisses dont nous avons connaissance aujourd'hui par les fragments 2-7.

\section{La troisième section du discours sur l'être}

Une fois démontrées les cinq (ou six) caractéristiques de l'être, que se passe-t-il ? Le passage qui suit (8.26-33) a l'apparence (mais seulement l'apparence) d'une vaste récapitulation : y est explicitement affirmée l'immobilité de l'être; répété que l'être n'a ni commencement ni fin (puisqu'il n'y a pas de sens à parler de naissance et de mort) ; affirmée sa complétude; précisé qu'il demeure lui-même en lui-même, qu’il gît en luimême, qu'il reste fixe (en somme, qu'il est dans une condition de stase parfaite); précisé en outre qu'il n'a besoin ni ne manque de quoi que ce soit, comme s'il éprouvait un sentiment de satisfaction. Le discours passe ainsi à un niveau supérieur, dans la mesure où l'être est maintenant objectivé, où il devient quelque chose que nous pouvons nous représenter, une entité sui generis (complète et statique, ne connaissant ni événements ni histoire).

En fait, dans ces vers, un nouveau mythe commence à prendre forme : le mythe d'un être totalement différent de l'univers qui est l'objet de nos expériences et de nos connaissances, un être qui, tout simplement, ne sert pas à «sauver les phénomènes » mais qui est traité désormais comme une réalité, comme une "chose » faite d'une certaine façon et dont on peut parler en connaissance de cause, dont on connait la ratio. Si les poètes ont pu parler comme de choses réelles du Minotaure, des centaures, des cyclopes et du sphinx, ce qui fait son apparition ici, c'est le mythe philosophique, un mythe qui ne se propose pas de satisfaire la curiosité d'auditeurs en mal d'images suggestives, mais d'être reçu comme un savoir de grande valeur sur une entité qui ne peut être pensée que comme une totalité anomale. La récapitulation apparente sert donc à faire un pas en avant décisif : non seulement commencer à se représenter cet être sur lequel porte tout le premier logos, mais aussi donner pour acquis que cette entité est connue et que l'on sait déjà beaucoup de choses sur son compte. 
Tout de suite après (v. 34-38a) est affirmée ouvertement la thèse que la pensée adhère à l'être, en mettant une certaine emphase à suggérer qu'une pensée bien conduite ne peut faire autrement qu'isoler l'être et le séparer des apparences (tout comme du non-être). L'idée sous-jacente pourrait être que la pensée est en proie à la contradiction tant qu'elle n'arrive pas à admettre que l'être a des caractéristiques complètement différentes des objets de l'expérience quotidienne, à savoir précisément les cinq (ou six) caractéristiques identifiées plus haut. Ce pas supplémentaire dépend étroitement de l'objectivation de l'être qui s'est produite aux vers 26-33. Suit, aux vers 38b-41, un corollaire introduit par $\tau \tilde{\omega}$, terme récurrent : si la pensée adhère à l'être et si, par conséquent, les mots aussi doivent être amenés à parler de l'être d'une façon qui ne soit pas impropre, alors les mots d'usage courant, naitre et mourir, ne pas être, se déplacer, changer, n'ont pas de pertinence, ce sont de simples noms. Est ainsi réitérée avec insistance la thèse que l'être est complètement étranger au monde de ce qui change ${ }^{31}$. Enfin les vers $42-49$, se fondant toujours sur l'objectivation réalisée auparavant, reprennent la thèse de la parfaite homogénéité et introduisent celle de la sphéricité de cette entité dénommée «être», avec probablement, pour corollaire, un état d'équilibre

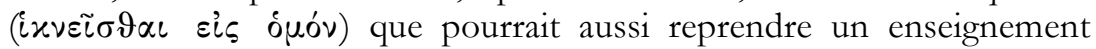
précis d'Anaximandre.

Dans l'ensemble, les vers 26-49 ont pour objectif la consolidation des résultats de la série des démonstrations fondamentales. La construction de la théorie devient une réalité, l'être s'objective, acquiert au moins quelques caractéristiques, on commence à comprendre ce qui est et ce qui n'est pas, en quels termes on peut en parler. C'est pourquoi nous pouvons dire que c'est dans cette troisième section que le résultat est atteint. La section initiale avait pour tâche de procéder à certains choix fondamentaux, la section la plus rigoureusement démonstrative avait pour tâche de construire la charpente de l'édifice, mais c'est seulement dans

31. Gemelli Marciano 2009 interprète ces vers de façon très tranchée: selon elle, dans les vers 38b-41 il est question, non pas de simples noms attribués par les mortels (donc de noms conventionnels, fruit de la croyance, chose bien différente de la façon correcte de se représenter l'être et d'en parler), mais de noms pertinents, légitimes, vrais. En conséquence, elle propose la traduction suivante : «sein Name wird 'alle Dinge' sein, / wie viele die Sterbliche angenommen haben, in der Überzugung, sie seien wahr usw.» (p. 23). Gemelli précise que les vers 38b-41 dissipent l'énigme, dans la mesure où ils nous font comprendre que le nom de l'être est «toutes choses» (p. 68 et 88) ; étant donné, cependant, les associations d'idées évoquées par brotoi, lesquelles se concrétisent aussi en B6.4 et B1.27, un changement de signe si spécifique (et si peu évident) - passage du manque de fiabilité à l'attibution de cette même fiabilité, donc conversion du négatif au positif aurait dû être dûment argumenté. 
cette troisième section que l'édifice commence à prendre forme et à se définir.

L'impression de fermer le cercle et d'arriver à une idée précise de cet être a cependant un prix : c'est la renonciation à toute hypothèse de raccordement entre cet être placé en hibernation et l'instabilité de ce qui apparait. Précisément, les vers qui suivent immédiatement (50-52) nous confirment l'intention de maintenir une discontinuité et une incommensurabilité très nettes entre la réalité vraie et la réalité apparente.

D'où la conclusion que s'appliquer à contourner l'obstacle et à rétablir à tout prix une quelconque continuité entre apparence et être n'a pas beaucoup de sens, non plus que réussir à nier que les deux domaines soient totalement étrangers l'un à l'autre. En effet, Parménide ne pouvait de façon plus drastique couper les ponts, séparer, créer une situation de totale incommunicabilité entre réalité vraie et réalité apparente. Prétendre forcer la situation jusqu'à raccorder ce que manifestement il ne veut raccorder d'aucune manière doit donc, malheureusement, être qualifiée d'arbitraire. La tour d'ivoire de l'être de Parménide peut certainement déconcerter, la division radicale de structures à compartiments étanches est propre, indubitablement, à susciter une sorte de malaise, mais nous ne pouvons pas ne pas constater que la volonté du poète-philosophe a été justement de couper les ponts entre l'être et tout autre type de réalité. Qu'il ait eu l'intention de définir son être comme une entité sans aucune relation avec autre chose (donc non modifiable, inattaquable et même, à sa façon, heureuse) est une donnée difficilement contestable.

\section{La grande césure (8.50-52) : un seul et même schéma théorique, deux jugements}

Il n'y a aucun doute que, en 8.50-52, Parménide conclut le discours sur l'être et aborde le second enseignement prévu. La déesse nous dit de la façon la plus claire que le logos sur l'être a désormais déployé toutes ses potentialités, qu'il est terminé et qu'il cède la place à un enseignement qui, bien que profondément différent de celui qui vient d'être délivré, mérite également d'être appris. Le passage a remarquablement stimulé la créativité exégétique des interprètes, mais une attention renouvelée à la façon dont Parménide assure la transition du premier au second logos est susceptible de nous révéler bien des choses. Pour commencer, j’observe que la section finale du fragment 8 propose un schéma général (repris au fragment 9) qui permet de se représenter le monde physique. Or ce schéma, qui tient en seulement cinq vers, s'ouvre (v. 53) et se ferme (v. 60 sq.) sur des jugements de valeur étonnamment différents bien que, de toute évidence, ils portent sur le même objet. J'espère montrer que 
leur co-présence à l'intérieur d'un discours continu n'est pas seulement digne de remarque mais aussi éclairante.

La première déclaration, de nature métadiscursive, est cohérente avec les déclarations de même nature qui ont trouvé place à la fin du fragment 1 et dans le cours du fragment $6:$ la façon dont raisonnent les mortels est infiniment inférieure au savoir professé par la déesse, parce qu'elle se fonde sur une erreur, à savoir attribuer une consistance au néant, traiter le néant, non comme tel, mais comme un ingrédient du réel. De manière analogue, nous lisons maintenant que, au lieu de construire une idée monolithique du réel, les mortels ont parlé de deux formes, qu'ils ont attribué des signes et des noms distincts à chacune, et que c'est là leur erreur ( façon de se représenter la réalité. On ne peut douter sérieusement du sens de ce que Parménide veut nous faire comprendre: dans la façon dualiste de se représenter le monde est à l'œuvre une grave erreur. Mais ce n'est là qu'une "demi-vérité ", car aussitôt après, et toujours à propos de cette vision dualiste du monde, sa déesse annonce qu'elle va prononcer une parole autorisée ( $\left.\varphi \alpha \tau i \zeta_{\omega}, \mathrm{B} 8.60\right)$ et proposer une représentation ordon-

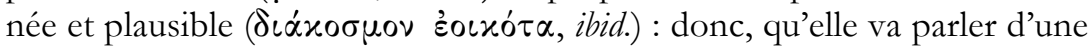
manière appropriée et fondée. Elle précise à cette occasion le but qu'elle veut atteindre : faire en sorte que le kouros se forge des opinions qui lui permettent d'affronter victorieusement les mortels (B8.61); que, dûment instruit, il puisse s'établir sur des positions nettement supérieures à celles des brotoi, même quand il aura à affronter l'univers des doxai (par exemple la cosmologie).

Comme on le voit, il se produit, en cinq vers seulement, un changement spectaculaire (et donc inattendu, problématique) dans l'attitude de la déesse. Le même enseignement est traité d'abord comme l'un des péchés capitaux, puis comme un savoir véritable qui n'est pas simplement compatible avec la haute dignité de la déesse qui va le dispenser, mais pleinement digne d'elle. En toute cohérence avec ce changement d'attitude, au fragment 10, la déesse ne se présente pas comme une chercheuse qui essaye de rendre compte des phénomènes tout en sachant qu'elle pourrait se tromper, mais comme la détentrice d'un savoir achevé : «je sais et je suis en mesure de t'expliquer, de te faire comprendre, de te faire pénétrer

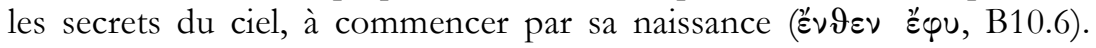
Ainsi, sous l'effet de ma parole, tu vas venir à savoir (Ë̌́n, B10.1).» Nous sommes donc en présence d'une déesse qui, sous nos yeux, change d'étalon de mesure, et nous constatons, non sans surprise, que Parménide lui-même donne une valeur positive à ce qui faisait jusque-là l'objet de jugements fortement négatifs (et, à une seule exception près, uniquement négatifs). 
D'où un corollaire que n'ont que rarement aperçu les interprètes : si les neuf derniers vers du fragment 8 sont dus à Parménide, alors il n'a pas pu ne pas se rendre compte du changement radical qui a lieu dans ces vers. Mais si Parménide change consciemment d'étalon de mesure, au point de presque oublier les jugements qu'il a lui-même formulés auparavant de manière si emphatique, il en découle une autre conséquence d'importance : il n'y a pas de sens à ouvrir le jeu (ou la bataille) des interprétations avant d'avoir reconnu l'importance exceptionnelle de cette très singulière décision de l'auteur, et d'avoir travaillé à l'interpréter. En effet, je pense que c'est là-dessus que l'on devrait avant tout s'interroger : sur l'anomalie représentée par un auteur qui, dans un premier temps, désavoue sans ménagement l'ensemble de son savoir doxastique, en vient ensuite à indiquer en quel point précis est vulnérable ce savoir mais, au bout de cinq vers à peine, se montre décidé à ignorer ces réserves de façon manifeste et à traiter aussi le second type de savoir comme un savoir authentique ( $c f$. عíøn, B10.1). Le fait n'est pas seulement sans exemple, il constitue également un passage crucial, parce qu'il est impossible que Parménide n'ait pas été conscient de frôler la contradiction. Mais s'il s'en est rendu compte, alors (a) il s'est aperçu qu'il se trouvait nier et affirmer la même chose, (b) il a compris qu'il n'était pas en mesure de résoudre le conflit et (c) n'a pas fait semblant de le résoudre d'une manière artificielle; pourtant (d) l'intolérance du premier type de savoir n'a pas suffi à le faire renoncer au second, et il s'est contenté de "limiter les dégâts » (e) en faisant coexister les deux types de savoir, (f) en usant de la possibilité de réduire au minimum les points de contact et d'interférence éventuelle entre eux. (g) L'organisation du poème répond à l'exigence d'assurer aux deux types de savoir une coexistence raisonnable. Je vais essayer d'expliquer les sept points que je viens d'indiquer.

(a) Comme on le sait, au nom du "principe de charité », la communauté scientifique a essayé de multiples façons de nier l'évidence d'un conflit sans issue entre le savoir sur l'être et le savoir sur le monde ; mais c'est Parménide lui-même qui, de manière emphatique, répétée, appuyée, a déclaré que le savoir sur le monde ne peut être mis sur le même plan que le savoir sur l'être - et même que l'on ne peut en reconnaître la légitimité tant que l'on se situe sur le plan de l'être. En d'autres termes, il a tout simplement exclu l'éventualité d'une issue théorique, doctrinale, élaborée à partir de la doctrine de l'être. Dont acte. Il est certes légitime et intéressant de chercher des issues que n'a pas envisagées Parménide, mais cela risque d'être un exercice purement spéculatif, dépourvu de pertinence exégétique.

(b) Si Parménide se voit dans l'obligation de rappeler que le savoir peri physeos ne reflète que le point de vue des mortels et que, même s'il est 
préservé et communiqué par la déesse, il ne peut être comparé au savoir sur l'être, c'est uniquement parce qu'il est face à un problème qu'il sait ne pas pouvoir résoudre. En d'autres termes, ayant échoué dans la tentative de concilier et de raccorder entre eux les deux types de savoir, il a fini par y renoncer.

(c) Le philosophe ose admettre que son enseignement dissimule à peine une question préjudicielle qu'il n'est pas en mesure de résoudre et que par conséquent il lui faut «vivre avec ». Dans ce choix d'admettre qu'entre les deux savoirs il y a un déséquilibre aussi insurmontable, on ne peut pas ne pas voir une marque d'honnêteté intellectuelle.

(d) Bien que, sous l'effet de la doctrine de l'être, le savoir sur le monde se voie privé de fondement, Parménide ne peut ni ne veut y renoncer. Il persiste en effet malgré tout à lui reconnaître une dignité propre, en tout cas par rapport aux opinions émises par d'autres intellectuels. La situation porte à penser que les doctrines présentées au cours du second logos sont le précieux fruit d'une première phase de ses recherches. En d'autres termes, il est vraisemblable que le "second savoir» du poème constitue ce que Parménide avait réussi à élaborer de meilleur dans les années qui ont précédé le moment où la réflexion sur l'être s'est frayé un chemin dans son esprit.

(e) Une fois admise l'existence définitive de facteurs d'incompatibilité entre les deux savoirs, une fois qu'on a reconnu que la légitimation

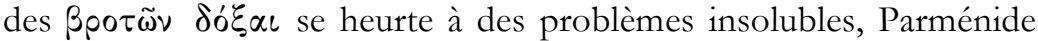
n'en affirme pas moins catégoriquement que ces opinions sont les meilleures (B8.61), qu'une personne cultivée en a besoin (B1.31), que ce savoir est sixós (B8.60), capable de «résister» à toute autre

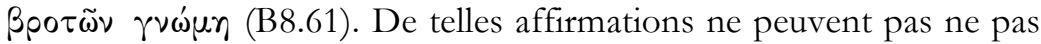
répondre à l'intention de rassurer ce même public qu'inquiétait la reconnaissance de graves facteurs d'incompatibilité entre les deux enseignements. Parménide n'hésite donc pas à faire les deux choses à la fois : admettre l'existence d'un problème grave et, aussitôt après, rassurer l'auditoire dans la mesure où son «second savoir » ne s'oppose pas à la doctrine de l'être mais uniquement au savoir qu'offrent d'autres œuvres peri physeos qui, à cette époque, circulaient dans l'œcoumène hellénique. Cette dernière circonstance nous aide à comprendre pourquoi il a mis un soin particulier à établir de multiples formes de discontinuité entre des sections du poème bien identifiées et bien différenciées, adoptant à chaque fois des "règles " particulières, au lieu de chercher à occulter les facteurs d'incompatibilité ou de donner à entendre une continuité et une homogénéité entre les deux savoirs. Il a réussi, de cette façon, non seulement à obtenir un ordre extraordinairement précis et réfléchi, mais aussi à isoler les deux types de sa- 
voir qu'il a développés, en réduisant au minimum les points de contact et les possibilités d'interférences entre les deux domaines.

(f) Le savoir sur l'être comporte un corollaire extrêmement gênant (l'être n'est pas le monde que nous connaissons, il n'offre pas de similitude avec lui, il n'aide pas à comprendre comment il se fait que le monde ait les caractéristiques que nous lui connaissons, il condamne à l'erreur la tendance à se représenter le monde comme un mélange de feu et de nuit, de lumière et d'obscurité), mais ce corollaire est incapable d'en générer d'autres, qui regarderaient le savoir «humain» sur le monde physique : il est donc sans interférence avec ce savoir. À son tour, le savoir sur le monde n'a rien à dire à propos du savoir sur l'être et peut facilement l'ignorer. En d'autres termes, aucun des deux savoirs n'a lieu d'interférer avec les contenus propres à l'autre. Ainsi se dessine, par conséquent, une possibilité concrète de supporter le malaise provoqué par le corollaire négatif.

(g) L'indifférence mutuelle de ces deux types de savoir virtuellement incompatibles résout le problème de leur coexistence. Il apparait ainsi que l'organisation du poème (son projet macro-rhétorique) est fonction de l'exigence de permettre cette coexistence, malgré l'impossibilité avouée d'expliquer le monde à partir de l'être et de fonder le savoir sur le monde à partir du savoir sur l'être.

Nous commençons ainsi à comprendre comment l'auteur lui-même s'est représenté le passage du premier au second type de savoir et d'enseignement, comment il a pu tolérer l'impossibilité de situer le discours sur le monde au sein du discours sur l'être.

\section{L'agencement du second logos}

C'est pour nous une chance insigne que d'avoir accès à la fois à des déclarations qui jettent le discrédit sur le savoir peri physeos (p. ex. B1.30) et sur l'é'́n de B10.1, car il y a là de quoi nous rassurer : ce n'est pas nous qui n'avons pas compris, c'est Parménide qui joue de la dissonance. Maintenant, en effet, Parménide, tout le premier, se désintéresse de l'être du non-être et se comporte comme s'il n'avait jamais écrit le premier logos, en particulier les vers 58-61 du fragment 8 et le fragment 9 en entier. En conséquence de quoi, nous pouvons essayer de remédier à un déséquilibre qui est une donnée de fait, mais nous ne sommes pas tenus de le faire et nous ne devons pas trop nous chagriner si nous échouons dans une entreprise que Parménide lui-même n'a pas conduite à son terme. En tout cas, nous ne pourrons comprendre le second logos aussi longtemps que nous l'envisagerons dans l'optique du premier. Par conséquent, en nous occupant du cosmos et du monde de la vie, nous aussi, nous devrons oublier l'être-sphère et l'inconsistance du néant. 
La nouvelle organisation de la communication, typique du second logos, se dessine avec une précision appréciable dans les fragments 10 et 11. Là en effet prend forme, de toute évidence, une nouveau prologue ou une formule de transition qui, au premier abord, consiste à donner la liste des sujets qui seront traités et des connaissances moyennement fiables que l'auditoire pourra acquérir par la suite : l'aither, le soleil, les phases de la lune, la voûte céleste, les étoiles et la voie lactée, la terre, c'est-àdire uniquement les corps célestes, et la terre dans la mesure où elle se rapporte aux autres corps célestes.

Il est aisé d'observer que ce savoir peri physeos est construit sur la base de principes d'organisation sans aucune continuité avec le savoir sur l'être. Ce qui est avant tout abandonné, c'est la structure démonstrative : la déesse ne déduit plus, elle n'argumente plus, mais elle affirme et, parfois, raconte. Les niveaux exemplaires de rigueur démonstrative qui caractérisent tout le développement sur l'être, des prémisses à la conclusion, sont une caractéristique exclusive de la section concernée ; on ne trouve rien de comparable dans le second logos. En outre, la déesse se présente comme détentrice d'un savoir accompli : « je sais et je suis en mesure de t'expliquer, de te faire comprendre, de te faire pénétrer les secrets du ciel,

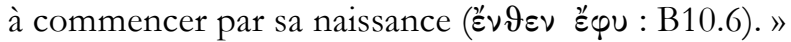

Une autre caractéristique formelle des fragments 10-11 est leur aptitude à fournir un embryon de sommaire des sujets qui seront traités. C'est comme un coup d'œil préliminaire sur l'ensemble de cette soussection, comme le fait remarquer aussi Simplicius en présentant le fragment 11. Le fait est significatif, si l'on considère que, non seulement à l'époque de Parménide mais aussi à celle d'Aristote, l'usage n'était pas encore apparu de faire précéder chaque développement d'un méta-texte servant de sommaire, et qu'Aristote se borne à offrir parfois des vues d'ensemble qui ne sont pas plus détaillées que celle-ci32. On peut donc dire que dans le peri physeos de Parménide apparaît pour la première fois l'idée d'un sommaire, idée qui n'a ni précédent ni d'autre postérité qu'Aristote, lequel n'essaye pas de faire en la matière quelque chose de plus élaboré ou formalisé, ce qui montre qu'il ne tient pas ce type de vue d'ensemble pour un critère d'excellence.

Puisqu'il n'est pas fait mention dans ces vers des problématiques typiques du monde de la vie, et en particulier de la reproduction humaine, ce prologue semble se rapporter exclusivement à la sous-section de caractère cosmologique. En outre, le fragment 19, d'allure indubitablement rétrospective, semble correspondre au prologue qui nous est parvenu.

32. On suppose que l'usage du sommaire est apparu à l'époque hellénistique, mais sans s'imposer immédiatement. 
Cela induit à supposer que ce dernier ne se réfère qu'à une seule partie de l'exposé peri physeos et qu'un prologue du même genre était réservé à l'exposé de caractère médical (lato sensu). On constate cependant que, si le rôle cosmique attribué à Anankè (B10.6) est de nature à confirmer tout cela, dans les fragments 12-13 (et en B11.13-14) apparaît une idée de fécondation rapportée au cosmos dans son ensemble, qui ne peut pas ne pas évoquer les anciens mythes. En outre, au fragment 12 apparaît inopinément un développement apparenté, du point de vue formel, à la tradition mytho-poétique (allure narrative, emploi du passé, indication du lieu où devrait résider une déesse, affirmations invérifiables qui ne sont que l'amplification de lieux communs...). Ce second ensemble d'indices $^{33}$ fait douter de la conjecture énoncée ci-dessus. Il est en effet au moins possible que Parménide ait voulu, en forçant un peu, donner à son exposé peri physeos une unité un peu artificielle en donnant au thème de la différence et de l'attraction sexuelle une portée cosmologique, en assimilant peut-être le couple feu-terre au couple mâle-femelle, de manière à établir une continuité entre les considérations sur l'éros cosmique et l'éros humain, biologique. Il ne semble pas y avoir d'indices qui permettent de dissiper ce doute.

Quoi qu'il en soit, la référence répétée à l'éros, la présence de passages peu éloignés de la logique du mythe et les aspects inévitablement paratactiques du savoir sur les corps célestes (au sens où le savoir sur la lune ne se déduit pas du savoir sur le soleil ou sur la voie lactée, pas plus que ce savoir ne peut se déduire du savoir sur la lune) confirment que le second logos était agencé d'une façon relativement voisine des peri physeos produits par d'autres sophoi et qu'il était donc, du point de vue de son statut épistémique et de ses formes d'exposition, profondément différent du savoir offert dans le premier logos. Ce qui parait cohérent avec l'insistance mise au début par la déesse à minimiser sa valeur cognitive.

Du point de vue de la dimension «architectonique» du poème, il n'est pas utile d'aborder les contenus du savoir peri physeos. Le moment est donc venu de consacrer un peu d'attention à la conclusion du poème (et de conclure). Pour ce qui concerne la fin du poème, je me bornerai à suggérer l'éventualité, déjà indiquée, que, une fois conclu le discours de la déesse, la narration reprenne brièvement son cours avec un remerciement du kouros et une sphragis peu éloignée de celle de Thucydide (cf. supra). Ce qui incite à le penser, c'est le soin avec lequel la déesse dit con-

33. Il faut en excepter le $\gamma \alpha \dot{\lambda} \alpha$ oủpávıov de B11.2. Il semble en effet que le «lait céleste » soit traité comme une partie de l'ensemble des corps célestes, et non comme un organisme auquel seraient attribuées des fonctions anthropomorphiques telles que «nourrir » les corps célestes ou les dieux. 
clure aussi bien le premier que le second logos (en B8.50 sqq. et en B19). Si la déesse sait introduire et conclure aussi bien l'ensemble de son discours que les parties dont il est constitué, il est pratiquement impensable que Parménide se soit refusé la possibilité d'ajouter quelques vers conclusifs, de manière à donner une valeur supplémentaire au prologue et à éviter qu'il ne reste sans conclusion, inachevé. Le goût avec lequel il s'applique à articuler le continuum du poème en une multiplicité de micro- et macrosections pousse à exclure l'éventualité d'une conclusion dépourvue du couronnement approprié, mais évidemment l'absence de données factuelles oblige à se borner à cette indication d'ordre général.

\section{Conclusion : un maître conscient de son savoir}

Dans son ensemble, le poème repose sur la juxtaposition de la construction d'une situation fantastique dans le proème, d'un édifice argumentatif abstrait dans le premier logos et d'un savoir peri physeos de nature plus discursive dans le second. Dans chacune de ces trois grandes sections s'affirment des façons profondément et visiblement différentes d'être poète et sophos. Parménide paraît ainsi maîtriser de multiples formes de raisonnement et d'exposition, dont chacune est introduite et utilisée au moment opportun, pour être ensuite abandonnée sans regret. Il excelle dans la construction d'un savoir organisé, disposé sur différents plans, articulé en parcours dont chacun arrive à se différencier des autres de façon très claire, et certainement pas pour des raisons purement esthétiques (comme par exemple la recherche de la variatio).

En même temps, il paraît décidé à faire vivre à son auditoire trois expériences incroyablement différentes: l'immersion dans une situation imaginaire, puis dans une structure démonstrative extrêmement rigide et soumise à une formalisation d'un niveau très élevé, et enfin dans un savoir peri physeos lui aussi créatif et précieux, mais d'une tout autre nature que tout ce qui le précède. L'horizon d'attente de l'auditoire est à chaque fois modelé de façon différente: il lui faut, dans la phase initiale, se laisser immerger dans une atmosphère fantastique peu différente de celle d'autres récits traditionnels et laisser grandir son attente des deux événements qui vont suivre; dans la deuxième phase, prendre confiance dans des abstractions, assimiler certaines idées (comme l'opposition entre être et non-être), puis toute une série d'inférences en cherchant à suivre le raisonnement, à comprendre et enfin à se convaincre que le raisonnement ne laisse rien à désirer ; dans la troisième phase, en revanche, on a l'impression que pour rassurer, entre autres choses, un auditoire nécessairement abasourdi, Parménide cherche à se placer sur le même plan que les nombreux traités peri physeos déjà en circulation (ne serait-ce que le poème didactique de Xénophane), de façon que la proposition d'autres ensei- 
gnements, même s'ils sont très innovants, use d'un langage relativement traditionnel et guère créatif. C'est en tout cas ce que les quelques vers qui subsistent incitent à penser pour ce qui concerne le second logos. Ce qui, du point de vue de la logique de la composition, n'a rien de surprenant.

Si telle fut l'articulation générale du poème, on peut comprendre que les intellectuels de l'époque aient été éblouis par le premier logos et seulement par lui, en raison non tant de l'emphase avec laquelle l'a introduit la déesse que de l'impression produite par les innovations inouïes qui le caractérisent du point de vue de sa construction et de son argumentation, alors qu'ils pouvaient sous-évaluer l'importance des théories présentées dans le second. Cette impression de se trouver face à un raisonnement doté d'une cohérence sans faille aide à comprendre pourquoi c'est la doctrine de l'être qui impressionna particulièrement les contemporains. 


\section{BIBLIOGRAPHIE}

Álvarez Salas, O. 2006 : Epicarmo e la sapienza presocratica, Diss. Univ., Turin, 2006.

Bollack, J. 2006 : Parménide : de l'étant au monde, Lagrasse, 2006 (Verdier poche).

Cerri, G. 1999 : Parmenide di Elea, Poema sulla natura, introduzione, testo, traduzione e note di -, Milan, 1999 (BUR classici greci e latini).

Cordero, N. L. et al. 2008 : Eleatica 2006 : Parmenide scienz̧iato?, a cura di L. Rossetti \& F. Marcacci, Sankt Augustin, 2008 (Eleatica, 1).

DiEls, H. 1897 : Parmenides Lehrgedicht, griechisch und deutsch, mit einem Anhang über griechische Thüren und Schlösser, Berlin, 1897 [2e éd., Sankt Augustin, 2003 (International PrePlatonic Studies, 3)].

GÁlveZ Garduño, R. 2008: «La poesía de Parménides: el arte del estilo ambiguo y desafiante, insinuador y sutil », Nova Tellus, 21 (2008), p. 55-90.

Gemelli Marciano, M. L. 2009 : Die Vorsokratiker. II, Parmenides, Zenon, Empedokles, Griechisch-lateinisch-deutsch, Auswahl der Fragmente und Zeugnisse, Übersetzung und Erläuterungen, Düsseldorf, 2009 (Sammlung Tusculum).

Granger, H. 2002 : «The Cosmology of Mortals », dans V. Caston \& D. Graham (éd.), Presocratic Pbilosophy: Essays in Honour of Alexander Mourelatos, AldershotBurlington, VT, 2002, p. 101-116.

MANSFELD, J. 1984 : «Mito, scienza, filosofia : una questione di origini », Quaderni di Storia, 20 (1984), p. 43-67.

MARCACCI, F. 2009 : Alle origini dell'assiomatica : gli Eleati, Aristotele, Euclide, Rome, 2009 (12008).

McKirahan, R. 2008 : Signs and Arguments in Parmenides B8, dans P. Curd \& D. Graham (éd.), The Oxford Handbook of Presocratic Philosophy, Oxford, 2008, p. 189-229.

RAMírez VIDAL, G. 2005 : La palabra y la flecha : análisis retórico de textos de la Grecia antigua, México, 2005.

Robbiano, C. 2006 : Becoming Being: on Parmenides' Transformative Philosophy, Sankt Augustin, 2006 (International Pre-Platonic studies, 5).

RosSETTI, L. 1994 : Strategie macro-retoriche : la «formattazione» dell'evento comunicazionale, Palerme, 1994 (Aesthetica Preprint, 41).

- 2008a : Introdurione [a N.-L. Cordero], dans Cordero et al. 2008, p. 7-24.

- 2008b : «La componente metacognitiva della filosofia e del filosofare», Giornale di Metafisica, n.s. 30 (2008), p. 3-30.

Thanassas, P. 2007 : Parmenides, Cosmos, and Being : a Pbilosophical Interpretation, Milwaukee, WS, 2007 (Marquette studies in philosophy, 57).

TulLi, M. 2000 : «Esiodo nella memoria di Parmenide», dans G. Arrighetti (éd.), Letteratura e riflessione sulla letteratura nella cultura classica: tradizione, erudirione, critica letteraria, filologia e riflessione filosofica nella produrione letteraria antica: atti del convegno, Pisa, 7-9 gingno 1999, Pise, 2000 (Biblioteca di studi antichi, 84), p. 65-81. 